\title{
RELATIONS BETWEEN THE CRITICAL POINTS OF A REAL FUNCTION OF $n$ INDEPENDENT VARIABLES* \\ BY
}

\author{
MARSTON MORSE
}

\section{INTRODUCTION}

In the main body of the paper the results and proofs are given for the general case of a real function of $n$ independent variables, and for a domain of definition of the given function that is very general from the point of view of analysis situs. For the purposes of an introduction one of the principal results will be given for the case where $n=2$. It must be stated, however, that the results for the general case differ tremendously from those for the case $n=2$. The results for the case $n \leqq 2$ can conveniently be given in geometric language as follows:

Let $R$ consist of the interior points of a finite, connected region of the $x, y$ plane, bounded by a finite number of distinct closed curves without multiple points of any sort, possessing at each point a tangent which turns continuously with movement of its point of contact. Denote the boundary of $R$ by $B$. Let $f(x, y)$ be a real function of $x$ and $y$ defined and continuous in $R$ and on $B$, and possessing continuous third order partial derivatives in $R$ taking on continuous boundary values on $B$. By the positive direction of the normal to $B$ will be understood the direction that leads across $B$ from points in $R$ to points not in $R$. It is assumed that the unilateral, directional derivative of $f(x, y)$ on the side of the inner half of the normal and in the positive direction along the normal is everywhere positive on $B$.

Let $z=f(x, y)$ be interpreted as a surface in ordinary euclidean $(x, y, z)$ space. By a critical point of $f(x, y)$ is meant a point in the $x, y$ plane corresponding to which the tangent plane to the surface $z=f^{\circ}(x, y)$ is parallel to the $x, y$ plane. In the neighborhood of such a critical point let $f(x, y)$ be expanded according to Taylor's formula in powers of differences of the $x$ 's and $y$ 's which vanish at the critical point, and with the remainder as a term of the third order. We hereby assume that in this expansion the discriminant of the terms of the second order is not zero.

From these assumptions it follows that the critical points are isolated and finite in number, and that they are of the two general types called

\footnotetext{
* Presented to the Society, December 28, 1923.
} 
in differential geometry elliptic and hyperbolic points. The elliptic critical points can be further subdivided into points at which $f(x, y)$ takes on a relative minimum, and those at which $f(x, y)$ takes on a relative maximum. Suppose that $f(x, y)$ has a relative minimum at $M_{0}$ different points, and a relative maximum at $M_{\mathbf{2}}$ different points, while the hyperbolic critical points are in number $M_{1}$. Suppose that $R$ and its boundary form a region of linear connectivity $R_{1}{ }^{*}$ With this understood we have the equality fundamental for the case $n=2$,

$$
M_{0}-M_{1}+M_{:}=2-R_{1} .
$$

From this it follows immediately that

$$
M_{1} \geqq M_{0}+R_{1}-2 .
$$

It should be noted that (a) does not follow from (b). The inequality (b) has been obtained by G. D. Birkhoff. $t$ Enunciated in slightly different form for the general case, he has called his result the "minimax principle" and he uses it to infer the existence of at least $M_{0}+R_{1}-2$ critical points of the hyperbolic type when the existence of $M_{0}$ points of relative minimum are known. He has carried out his work for the general case of real, analytic function of $n$ real variables and applied his result to the theory of dynamical systems. Upon reading Birkhoff's paper it occurred to the author that inasmuch as there are $n+1$ different kinds of critical points possible (in a sense to be defined later) there ought to be relations analogous to (b) involving the numbers of critical points of all kinds and not simply of just two kinds. With a proper interpretation of $M_{1}$ and $R_{1}$, (b) however will serve to state Birkhoff's "minimax principle" in any number of dimensions.

The author has replaced the inequality (b) by an equality involving all of the $n+1$ possible types of critical points, and in addition all of the connectivity numbers of the $n$-dimensional region of definition of the given function (Theorems 8 and 9). Further, a set of $n$ additional inequality relations have been discovered. The author ventures without proof the opinion that these relations, or relations derivable from them, are all the the relations there are between the numbers involved.

* Oswald Veblen, Analysis Situs, The Cambridge Colloquium, Part 2, p. 50, § 28.

We shall have occasion in the future as well as here to make use of the terms of analysis situs. They will be used in the senses in which they are defined in the work just cited. In the remainder of this paper reference to this work will be indicated by the letter $\mathrm{V}$.

$\dagger$ Dynamical systems with two degrees of freedom, these Transactions, vol. 18 (1917), p. 240. 
Note. For the case $n=2$ the author has been able to outline an alternative proof of one of the relations between the critical points by making use of the Kronecker characteristic of the given function and its partial derivatives. That the Kronecker theory as developed by Kronecker (Werke, vol. I, pp. 175-226, and vol. II, pp. 71-82) will not suffice in general to obtain the results of this paper is evident from the fact that Kronecker distinguishes between the critical points according to the sign of the hessian of the function, while in this paper both in the proofs and in the results there is an essential distinction between the $n+1$ types of critical points. Further, in Kronecker no reference is made to anything like the connectivity numbers of the regions in which the critical points are being studied.*

\section{The Function $f\left(x_{1}, x_{2}, \cdots, x_{n}\right)$}

1. The function $f\left(x_{1}, x_{2}, \cdots, x_{n}\right)$ and its critical points. Let $\left(x_{1}, x_{2}, \cdots, x_{n}\right)$ be the coördinates of any point $(X)$ in a euclidean space of $n$ dimensions. In a finite part of this space let there be given an $n$-dimensional region $\Sigma$. The set of interior points of $\Sigma$ will be denoted by $R$. At every point of $R$ let there be defined a real, single-valued, continuous function of the $x$ 's, namely $f\left(x_{1}, x_{2}, \cdots, x_{n}\right)$. Suppose further that in $R$ this function possesses continuous partial derivatives with respect to each of the $x$ 's of at least the first and second orders.

A point of $R$ at which all of the first partial derivatives of $f$ vanish will be called a critical point of $f$. Any other point of $R$ will be called an ordinary point of $f$. The value of $f$ at a critical point will be termed a critical value of $f$. As a further assumption regarding $f$ we will suppose that in the neighborhood of each critical point $f$ possesses continuous third partial derivatives. Let there be given a critical point of $f$ at which $f$ takes on the value $c$. For simplicity suppose this critical point at the origin. In the neighborhood of the origin, $f$ can be represented with the aid of Taylor's formula in the form

$$
\begin{aligned}
f^{\prime}\left(x_{1}, x_{i}, \cdots, x_{n}\right)-c=\sum_{i j} \alpha_{i j} x_{i} x_{j}+r\left(x_{1}, x_{2}, \cdots, x_{n}\right) \\
\left(i, j=1,2, \cdots, n ; \alpha_{i j}=\alpha_{j i}\right),
\end{aligned}
$$

where the terms of first order are missing because the origin is a critical point, where the terms of second order are represented as a symmetric quadratic form in the $n x$ 's with constant coefficients, and where the remainder $r\left(x_{1}, x_{2}, \cdots, x_{n}\right)$ is included as a term of the third order. In

\footnotetext{
* For closely connected results in the case $n=2$ see a paper by H. Poincaré, Journal de Mathématiques, ser. 3, vol. 7 (1881), pp. 375-421.
} 
any representation such as this of the function $f$ in the neighborhood of a critical point, it is hereby assumed that the terms of the second order constitute a non-singular quadratic form. Analytically this means that the determinant

$$
\left|\alpha_{i j}\right| \neq 0
$$

It is a consequence of this last restriction upon $f$ that in a sufficiently small neighborhood of any critical point there is no other critical point. For, if the partial derivative of $f$ with respect to $x_{i}$ be denoted by $f_{i}$ $(i=1,2, \cdots, n)$, each critical point of $f$ is a solution of the equations

$$
f_{1}=f_{2}=\cdots=f_{n}=0 \text {. }
$$

Now the jacobian of the $n$ functions $f_{i}$ with respect to the $n$ variables $x_{i}$ at the critical point we are here considering, namely the origin, vanishes only with the determinant $\left|\alpha_{i j}\right|$ which we have assumed not zero. It follows from the theory of implicit functions that the system of equations (3) has no other solution in the neighborhood of the origin than the origin itself. Thus there are no other critical points in the neighborhood of the origin.

A consequence of the result just obtained is that in any closed region $S$ all of whose points are points of $R$, there are at most a finite number. of critical points of the given function. For if there were an infinite number of critical points in $S$, by the Weierstrass cluster point theorem, these critical points would have at least one cluster point, which, since $S$ is closed, would belong to $S$, and hence would be a point of $R$. At each of these critical points all of the first partial derivatives of $f$ would vanish, and since these partial derivatives are assumed continuous at every point in $R$, they would all be zero at the cluster point. Thus the cluster point would also be a critical point contrary to the result of the preceding paragraph that the critical points of $f$ are isolated.

2. Certain preliminary lemmas. Lemmas 1 and 2 of this section are important aids in later proofs. They refer to a function $F\left(x_{1}, x_{2}, \cdots, x_{n}\right)$, real, single-valued, and continuous in the neighborhood of the origin (written $N$ ). To aid in proving Lemma 1, results (A), (B), and (C) will first be established.

(A) If $F\left(x_{1}, x_{\mathbf{z}}, \cdots, x_{n}\right)$ possesses continuous first partial derivatives in $N$, then in $N$

(4) $\quad F\left(x_{1}, x_{2}, \cdots, x_{n}\right)=F\left(0, x_{2}, x_{3}, \cdots, x_{n}\right)+a\left(x_{1}, x_{2}, \cdots, x_{n}\right) x_{1}$

where $a\left(x_{1}, x_{2}, \cdots, x_{n}\right)$ is single-valued and continuous in $N$. 
A function $a\left(x_{1}, x_{2}, \cdots, x_{n}\right)$ readily seen to satisfy the requirements of (A) is defined by the equations

$$
\begin{aligned}
\text { (5) } \quad a\left(x_{1}, x_{2}, \cdots, x_{n}\right) & =\frac{F\left(x_{1}, x_{2}, \cdots, x_{n}\right)-F\left(0, x_{2}, \cdots, x_{n}\right)}{x_{1}}, x_{1} \neq 0 \\
\text { (6) } \quad a\left(0, x_{2}, x_{3}, \cdots, x_{n}\right) & =F_{1}\left(0, x_{2}, \cdots, x_{n}\right)
\end{aligned}
$$

wherc $F_{i}$ will be taken to mean the partial derivative of $F$ with respect to $x_{i}(i=1,2, \cdots, n)$.

(B) If $F\left(x_{1}, x_{2}, \cdots, x_{n}\right)$ possesses continuous second partial derivatives in $N$, then in $N$

$$
\begin{aligned}
F\left(x_{1}, x_{2}, \cdots, x_{n}\right)=F\left(0, x_{2}, \cdots, x_{n}\right) & +F_{1}\left(0, x_{2}, \cdots, x_{n}\right) x_{1} \\
& +b\left(x_{1}, x_{2}, \cdots, x_{n}\right) x_{1}^{2}
\end{aligned}
$$

where $b\left(x_{1}, x_{2}, \cdots, x_{n}\right)$ is single-valued and continuous in $N$.

A function $b\left(x_{1}, x_{2}, \cdots, x_{n}\right)$ readily seen to satisfy the requirements of (B) is defined by the equations

(8) $b\left(x_{1}, x_{2}, \cdots, x_{n}\right)$

$$
=\frac{F\left(x_{1}, x_{2}, \cdots, x_{n}\right)-F\left(0, x_{2}, \cdots, x_{n}\right)-F_{1}\left(0, x_{2}, \cdots, x_{n}\right) x_{1}}{x_{1}^{2}}, x_{1} \neq 0,
$$

(9) $b\left(0, x_{2}, \cdots, x_{n}\right)=\frac{F_{11}\left(0, x_{2}, \cdots, x_{n}\right)}{2}$,

where $F_{i j}$ will be taken to mean the second partial derivative of $F$ with respect to $x_{i}$ and $x_{j}(i, j=1,2, \cdots, n)$.

(C) If $F\left(x_{1}, x_{2}, \cdots, x_{n}\right)$ possesses continuous first partial derivatives in $N$, then in $N$

(10) $F\left(x_{1}, x_{2}, \cdots, x_{n}\right)=F(0,0, \cdots, 0)+\sum_{i} a_{i}\left(x_{1}, x_{2}, \cdots, x_{n}\right) x_{i} \quad(i=1,2, \cdots, n)$, where each $a_{i}$ can be so chosen as to be continuous in $N$.

From (A) it follows that (C) is true for $n=1$. Following the method of mathematical induction the truth of (C) is now assumed for the case of $n-1$ independent variables. Whence

$$
F\left(0, x_{2}, \cdots, x_{n}\right)=F(0,0, \cdots, 0)+\sum_{i} b_{i}\left(x_{2}, \cdots, x_{n}\right) x_{i} \quad(i=2, \cdots, n)
$$

where each $b_{i}$ is continuous in $N$. If $F\left(0, x_{2}, \cdots, x_{n}\right)$ in (4) be replaced by the right hand member of (11) the result (C) follows directly. 
Lemma 1. If $F\left(x_{1}, x_{2}, \cdots, x_{n}\right)$ possesses continuous second partial derivatives in $N$, and vanishes with its first partial derivatives at the origin, then in $N$

$$
F\left(x_{1}, x_{2}, \cdots, x_{n}\right)=\sum_{i j} a_{i j}\left(\grave{x}_{1}, x_{2}, \cdots, x_{n}\right) x_{i} x_{j} \quad(i, j=1,2, \cdots, n)
$$

where each function $a_{i j}$ can be so chosen as to be continuous in $N$.

For $n=1$ (B) shows that Lemma 1 is true. If Lemma 1 be assumed to be true for the case of $n-1$ independent variables, then

$$
F\left(0, x_{2}, \cdots, x_{n}\right)=\sum_{i j} b_{i j}\left(x_{2}, \cdots, x_{n}\right) x_{i} x_{j} \quad(i, j=2,3, \cdots, n)
$$

where each $b_{i j}$ is continuous in $N$. If $F\left(x_{1}, x_{2}, \cdots, x_{n}\right)$ satisfies the hypotheses of this lemma, $F_{1}\left(0, x_{2}, \cdots, x_{n}\right)$ will satisfy the hypotheses (C) so that

$$
F_{1}\left(0, x_{2}, \cdots, x_{n}\right)=\sum_{i} c_{i}\left(x_{2}, \cdots, x_{n}\right) x_{i} \quad(i=2,3, \cdots, n)
$$

where each $c_{i}$ is continuous in $N$. If now in (7) $F\left(0, x_{2}, \cdots, x_{n}\right)$ and $F_{1}\left(0, x_{2}, \cdots, x_{n}\right)$ be replaced by their values from (13) and (14) respectively, then Lemma 1 follows as stated.

To prove Lemma 2 , the following results, $\left(\mathrm{A}^{\prime}\right),\left(\mathrm{B}^{\prime}\right)$, and $\left(\mathrm{C}^{\prime}\right)$, will first be proved.

(A') If $F$ possesses continuous second partial derivatives in $N$ then the function $a\left(x_{1}, x_{2}, \ldots, x_{n}\right)$ of $(\mathrm{A})$ as defined by (5) and (6) has continuous first partial derivatives in $N$.

It follows from (5) that $a\left(x_{1}, x_{2}, \ldots, x_{n}\right)$ has continuous first partial derivatives in $N$ if $x_{1} \neq 0$. For $x_{1}=0$ the partial derivatives of $a\left(x_{1}, x_{2}, \ldots, x_{n}\right)$ with respect to $\left(x_{2}, x_{3}, \cdots, x_{n}\right)$ exist, as follows from (6). For $x_{1} \neq 0$ it follows from Taylor's formula that

(15) $a\left(x_{1}, x_{2}, \cdots, x_{n}\right)=F_{1}\left(0, x_{2}, \cdots, x_{n}\right)+\frac{F_{11}\left(\theta x_{1}, x_{2}, \cdots, x_{n}\right) x_{1}}{2}, 0<\theta<1$.

From (15) and (6) and the definition of a derivative it follows that the partial derivative

$$
a_{x_{1}}\left(0, x_{2}, \cdots, x_{n}\right)=\frac{F_{11}\left(0, x_{2}, \cdots, x_{n}\right)}{2} .
$$

To prove $a_{x_{1}}\left(x_{1}, x_{2}, \cdots, x_{n}\right)$ continuous for $x_{1}=0$, observe that from (5) for $x_{1} \neq 0$ it follows that

$$
a_{x_{1}}=\frac{x_{1} F_{1}\left(x_{1}, x_{2}, \cdots, x_{n}\right)-\left[F\left(x_{1}, x_{2}, \cdots, x_{n}\right)-F\left(0, x_{2}, \cdots, x_{n}\right)\right]}{x_{1}^{2}},
$$

which by two applications of Taylor's formula becomes 


$$
\begin{aligned}
& x_{1}\left[F_{1}\left(0, x_{2}, \cdots, x_{n}\right)+F_{11}\left(\theta x_{1}, x_{2}, \cdots, x_{n}\right) x_{1}\right] \\
& x_{1}^{2} \\
& -\frac{F_{1}\left(0, x_{2}, \cdots, x_{n}\right) x_{1}+\frac{F_{11}\left(\theta^{\prime} x_{1}, x_{2}, \cdots, x_{n}\right) x_{1}^{2}}{2}}{x_{1}^{2}} \\
& =F_{11}\left(\theta x_{1}, x_{2}, \cdots, x_{n}\right)-\frac{F_{11}\left(\theta^{\prime} x_{1}, x_{2}, \cdots, x_{n}\right)}{2}, 0<\theta<1,0<\theta^{\prime}<1,
\end{aligned}
$$

whence

$$
\lim _{x_{1} \rightarrow 0} a_{x_{1}}\left(x_{1}, x_{2}, \cdots, x_{n}\right)=\frac{F_{11}\left(0, x_{2}, \cdots, x_{n}\right)}{2}
$$

and $a_{x_{1}}$ is thereby proved continuous in $N$ without exception.

To show that $a_{x_{2}}\left(x_{1}, x_{2}, \cdots, x_{n}\right)$ is continuous in $N$ for $x_{1}=0$ observe that from (5) for $x_{1} \neq 0$ it follows that

$$
a_{x_{2}}\left(x_{1}, x_{2}, \cdots, x_{n}\right)=\frac{F_{2}\left(x_{1}, x_{2}, \cdots, x_{n}\right)-F_{2}\left(0, x_{2}, \cdots, x_{n}\right)}{x_{1}},
$$

which by Taylor's formula gives

$$
a_{x_{2}}\left(x_{1}, x_{2}, \cdots, x_{n}\right)=F_{21}\left(\theta_{1} x_{1}, x_{2}, \cdots, x_{n}\right), \quad 0 \div \theta \cdots 1, x_{1} \neq 0,
$$
while from (6)

$$
a_{x_{2}}\left(0, x_{2}, \cdots, x_{n}\right)=F_{12}\left(0, x_{2}, \cdots, x_{n}\right) .
$$

From (16) and (17) it follows that $a_{x_{2}}$ is contintous in $N$ even when $x_{1}=0$. The remaining partial derivatives of $a\left(x_{1}, x_{2}, \cdots, x_{n}\right)$ are similarly seen to be continuous in $N$.

$\left(\mathrm{B}^{\prime}\right)$ If $F$ possesses continuous thivd partial derivatives in $N$, then the function $b\left(x_{1}, x_{2}, \cdots, x_{n}\right)$ of (B) as defined by (8) and (9) possesses contimuous first partial derivatives in $N$.

The proof of $\left(\mathrm{B}^{\prime}\right)$ is similar to that of $\left(\mathrm{A}^{\prime}\right)$.

$\left(\mathrm{C}^{\prime}\right)$ If $F$ possesses continuous second partial derivatives in $N$ then the functions $a_{i}\left(x_{1}, x_{2}, \cdots, x_{n}\right)$ of $(\mathrm{C})$ can be so chosen as to possess continuous first partial derivatives in $N$.

A review of the proof of $(\mathrm{C})$ with $\left(\mathrm{A}^{\prime}\right)$ in mind will show the truth of $\left(\mathrm{C}^{\prime}\right)$.

Lемма 2. If $F$ possesses continuous third partial derivatives in $N$, and vanishes with its first partial derivatives at the origin, then $F$ can be represented in the form

$$
F\left(x_{1}, x_{2}, \cdots, x_{n}\right)=-\sum_{i j} a_{i j} x_{i} x_{j} \quad(i, j=1,2, \cdots, n)
$$

where each $a_{i j}$ possesses continuous first partial derivatives in $N$. 
A review of the proof of Lemma 1 with $\left(\mathrm{A}^{\prime}\right),\left(\mathrm{B}^{\prime}\right)$ and $\left(\mathrm{C}^{\prime}\right)$ in mind will show the truth of Lemma 2 .

Lemma 3. The functions $a_{i j}\left(x_{1}, x_{2}, \ldots, x_{n}\right)$ of Lemmas 1 and 2 are such that at the origin

$$
a_{i j}+a_{j i}=F_{i j} \text {. }
$$

Under the hypotheses of Lemmas 1 and 2

$$
F\left(x_{1}, x_{2}, \cdots, x_{n}\right)=\frac{\sum_{i j} F_{i j}\left(\theta_{1} x_{1}, \theta_{2} x_{2}, \cdots, \theta_{n} x_{n}\right) x_{i} x_{j}}{2}, 0<\theta_{i}<1 .
$$

Hence from (20) and (18)

$$
\sum_{i j} a_{i j} x_{i} x_{j}=\frac{\sum_{i j} F_{i j}\left(\theta_{1} x_{1}, \theta_{2} x_{2}, \cdots, \theta_{n} x_{n}\right) x_{i} x_{j}}{2} .
$$

Let $\left(y_{1}, y_{2}, \cdots, y_{n}\right)$ be any point in $N$, and let $r$ be a positive constant so small that $\left(r y_{1}, r y_{2}, \ldots, r y_{n}\right)$ is a point in $N$ for any choice of $\left(y_{1}, y_{2}, \cdots, y_{n}\right)$ in $N$. In (21) set $x_{i}=r y_{i}$ and in the resulting equation cancel the factor $r^{2}$ from both members. There will then result the equation

(22) $\sum_{i j} a_{i j}\left(r y_{1}, r y_{2}, \cdots, r y_{n}\right) y_{i} y_{j}=\frac{\sum_{i j} F_{i j}\left(r \theta_{1} y_{1}, r \theta_{2} y_{2}, \cdots, r \theta_{n} y_{n}\right) y_{i} y_{j}}{2}$.

The two members of (22) possess limits as $r$ approaches zero, and these limits are equal, giving

$$
\sum_{i j} a_{i j}(0,0, \ldots, 0) y_{i} y_{j}=\frac{\sum_{i j} F_{i j}(0,0, \ldots, 0) y_{i} y_{j}}{2} .
$$

Equation (23) is an identity in the $y$ 's. Hence the coefficients of corresponding powers are equal. The equation (19) follows directly and the lemma is proved.

3. Different types of critical points. Let there be given a critical point of $f$. At this critical point let $c$ be the value of $f$. If this critical point be supposed to lie at the origin, it follows from Lemma 2 that in the neighborhood of the origin the difference $f-c$ can be represented in the form

$$
f\left(x_{1}, x_{2}, \cdots, x_{n}\right)-c=\sum_{i j} a_{i j}\left(x_{1}, x_{2}, \cdots, x_{n}\right) x_{i} x_{j} \quad(i, j=1,2, \cdots, n)
$$

where $a_{i j}\left(x_{1}, x_{2}, \cdots, x_{n}\right)$ is a single-valued, continuous function of the $x$ 's in a sufficiently small neighborhood of the origin, provided with continuous 
first partial derivatives with respect to each of the $x$ 's. Further without any loss of generality, we can suppose that

$$
a_{i j}=a_{j i} \text {. }
$$

If we set $a_{i j}(0,0, \cdots, 0)=\alpha_{i j}$, according to Lemma 3 , we will have at the origin

$$
2 \alpha_{i j}=f_{x_{i} x_{j}} .
$$

Equation (26) shows that the constants $\alpha_{i j}$ appearing here are the same as the constants $\alpha_{i j}$ used in (1). In $\S 1$ we assumed that $\left|\alpha_{i j}\right| \neq 0$. We can conclude that

$$
\left|a_{i j}\right| \neq 0,
$$

in a sufficiently small neighborhood of the origin.

According to the well known theory of quadratic forms a non-singular quadratic form in $n$ variables $\left(x_{1}, x_{2}, \ldots, x_{n}\right)$, by a real, non-singular, linear transformation of $\left(x_{1}, x_{2}, \cdots, x_{n}\right)$ into $n$ new variables $\left(y_{1}, y_{2}, \cdots, y_{n}\right)$, can be reduced to a quadratic form

$$
-y_{1}^{2}-y_{2}^{2}-\cdots-y_{k}^{2}+y_{k+1}^{2}+\cdots+y_{n}^{2}
$$

where $k$ is one of the integers from 0 to $n$, inclusive. ${ }^{*}$ An examination of the Lagrange method of making this reduction shows that it can be carried through in the case where the coefficicnts $a_{i j}$ are not constants, by formally following the method used for the case where the coefficients $a_{i j}$ are constants, writing down the Lagrange transformation at each stage with the variable coefficients $a_{i j}$ substituted for the constant coefficients $a_{i j}$ of the ordinary theory, provided at each stage at least one of the coefficients $a_{i i}$ is not zero at the origin. If at any stage all of the coefficients $a_{i i}$ are zero at the origin, at least one of the remaining coefficients, say $a_{r s}$, will not be zero at the origin since $\left|\alpha_{i j}\right| \neq 0$. If the preliminary transformation

$$
\begin{aligned}
& x_{r}=z_{r}-z_{s}, \\
& x_{s}=z_{r}+z_{s}, \\
& x_{i}=z_{i}
\end{aligned}
$$

be made the resulting coefficients of $z_{r r}$ and $z_{s s}$ will not be zero at the origin, and the Lagrange type of transformation can then be used. The transformation will of course be restricted to a neighborhood of the origin for which $\left|a_{i j}\right| \neq 0$ and such that each $a_{i j}$ by which it has been necessary

* Bôcher, Introduction to Higher Algebra, p. 131. 
to divide shall not be zero. If the given critical point is not at the origin a proper translation of the space of the $n x$ 's will bring the critical point to the origin. The above method of reduction of the function $f$ will then give the following lemma.

Lemia 4. If $f\left(x_{1}, x_{2}, \cdots, x_{n}\right)$ takes on the value $c$ at a critical point $\left(x_{1}^{0}, x_{2}^{0}, \cdots, x_{n}^{0}\right)=\left(X_{0}\right)$ then there exists a real transformation of the form

$$
y_{i}=\sum_{j}\left(c_{i j}\left(x_{j}-x_{j}^{0}\right), \quad c_{i j} \mid \neq 0 \quad(i, j=1,2, \cdots, n)\right.
$$

under which

$$
f\left(x_{1}, x_{2}, \cdots, x_{n}\right) \cdots c=y_{1}^{2}-y_{2}^{2}-\cdots y_{k}^{2}+y_{k+1}^{2}+\cdots+y_{n}^{2}
$$

where each $c_{i j}$ is a continuous function of the $x$ 's provided with continuous first partial derivatives in a sufficiently small neighborhood of $\left(X_{0}\right)$, and where $l_{k}$ is some integer from 0 to $n$, inclusive.

The integer $k$ is called the type of the given critical point. The number of critical points of the $k$ th type under boundary conditions to be introduced presently will be shown to be finite and will be denoted by $m_{k}$. It is the purpose of this paper to obtain the relations between the number's $m_{k}$ under various types of boundary conditions.

The jacobian of the functions $\left(y_{1}, y_{2}, \cdots, y_{n}\right)$ of (29) with respect to the variables $\left(x_{1}, x_{2}, \cdots, x_{n}\right)$ reduces at $\left(X_{0}\right)$ to the value of $c_{i j}$ at $\left(X_{0}\right)$. Thus this jacobian is not zero at $\left(X_{0}\right)$. It follows that the tronsformation (29) is one-to-one in the neighborhood of the given critical point.

4. The boundary conditions $\alpha$. In order to proceed further it is necessary to make some assumptions regarding boundary conditions. The first boundary conditions that we introduce are, strictly speaking, not boundary conditions alone. They will be shown later to lead to results depending upon boundary conditions in a strict sense. These first boundary conditions which we consider arc introduced because of their simplicity.

The boundary conditions a will be considered as fulfilled if among the $(n-1)$-dimensional spreads

$$
f\left(x_{1}, x_{2}, \cdots, x_{n}\right)==\text { const. }
$$

there exists one, say A, with the following properties:

I. $A$ is a closed manifold;

II. A forms the complete boundary of a portion of space, $S$, including $A$. all of whose points are points of $h$;

III. Each critical point of $f$ is an interior point of $S$;

IV. The value of $f$ on $A$ is greater than the value of $f$ at any interior. point of $S$. 
The region $S$ as defined in the boundary conditions $\alpha$ is a closed region containing all of the critical points of $f$. It follows from the results of $\S 1$ that there are at most a finite number of critical points of $f$. According to condition III there is no critical point of $f$ on $A$.

5. The complex of points satisfying $f\left(x_{1}, x_{2}, \cdots, x_{n}\right) \leqq c$. According to the boundary condition IV the absolute maximum of $f$ in $S$ is taken on at each point on the boundary of $S$ and at no other point of $S$. If any absolute minimum point of $f$ in $S$ be joined by a continuous curve to some boundary point of $S$, every constant $c$ between the absolute minimum $m$ of $f$ in $S$ and the absolute maximum $M$ of $f$ in $S$ will be taken on by $f$ at some point in $S$ on this curve. Let $c$ be any such constant between $m$ and $M$, not a critical value of $f$. The set of points $\left(x_{1}, x_{2}, \cdots, x_{n}\right)=(X)$, lying in $S$ and satisfying

$$
f\left(x_{1}, x_{2}, \cdots, x_{n}\right)=c
$$

according to the choice of $c$, are all ordinary points of $f$. This set of points is closed since $f$ is continuous. Its points are all interior points of $S$ since the value of $f^{\prime}$ on $S^{n}$ s boundary is $M$. If $\left(X_{0}\right)$ be any point on (31), at least one of the partial derivatives of $f$, say $f_{x_{1}}$, will not be zero at $\left(X_{0}\right)$. It follows that the points $\left(x_{1}, x_{2}, \cdots, x_{n}\right)$ which satisfy (31) in the neighborhood of $\left(X_{0}\right)$ can be represented in the form

$$
x_{1}=h\left(x_{2}, x_{3}, \cdots, x_{n}\right)
$$

where $h\left(x_{2}, x_{3}, \cdots, x_{n}\right)$ is a single-ralued function of its arguments provided with continuous partial derivatives up to the second order all in the neighborhood of $\left(X_{0}\right)$. Geometrically this means that the manifold of points satisfying (31) possesses at each point of (31) a hypertangent plane which turns continuously as the point of tangency varies continuously on (31). The manifold (31) thus has no singular points.

The set of points $(X)$ in $S$ satisfying

$$
f \leqq
$$

will make up an $n$-dimensional region, say $C_{n}$. In particular $C_{n}$ will include all of the points neighboring any absolute minimum of $f$. Its points are all interior points of $S$, and its boundary obviously consists of the points $(X)$ satisfying (31). It can be shown that $C_{n}^{\prime}$ is an $n$-dimensional complex in the technical sense of analysis situs.* In a proof of this the same sort of considerations would enter as enter in connection with the so called "regular regions" in the plane.t

* See V, loc. cit., Chap. 3.

† W. F. Osgood, Lehrbuch der Funktionentheorie, p. 179. 
The variation of the connectivity numbers $R_{0}, R_{1}, \cdots, R_{n}$ of $C_{n}$ with variations of $c$ will now be studied. To that end it will be convenient to consider first the trajectories orthogonal to the manifolds $f=$ const.

6. The trajectories orthogonal to the manifolds $f=$ const. In studying the trajectories orthogonal to the manifolds $f=c$ the neighborhoods of critical points of $f$ will be excluded from consideration. The trajectories orthogonal to the manifolds $f=c$ at ordinary points of $f$ might be defined as those curves along which the differentials $d x_{1}, d x_{2}, \ldots, d x_{n}$ at any point are proportional to the partial derivatives $f_{1}, f_{2}, \cdots, f_{n}$ at that point, where $f_{i}$ is used to denote the partial derivative of $f$ with respect to $x_{i}$ $(i=1,2, \cdots, n)$. Two trajectories are considered as the same if they consist of the same points $\left(x_{1}, x_{2}, \cdots, x_{n}\right)$. It is thus explicitly pointed out that a difference of parametric representative alone does not, according to this convention, constitute a difference in the trajectories.

At any ordinary point of $f$ at least one of the partial derivatives of $f$ is not zero. Let $(P)$ be an ordinary point of $f$ and suppose that at $(P)$ the partial derivative $f_{m} \neq 0$. The trajectories passing through the points neighboring $(P)$ will be solutions of the differential equations

$$
\frac{d x_{i}}{d x_{m}}=\frac{f_{i}}{f_{m}},
$$

where $i$ takes on all the integers from 1 to $n$ except $m$. According to the fundamental existence theorems for ordinary differential equations there is one and only one trajectory through each point of a sufficiently small neighborhood of $(P)$.

A set of differential equations will now be introduced in which the independent variable is not $x_{m}$ but a parameter $\boldsymbol{\tau}$, but whose solutions also represent orthogonal trajectories. The equations are

$$
\frac{d x_{i}}{d \tau}=r f_{i} \quad(i=1,2, \cdots, n)
$$

where

$$
r=\frac{1}{f_{1}^{2}+f_{2}^{2}+\cdots+f_{n}^{2}}
$$

The particular form of dependence of the $x$ 's upon $\tau$ which is required by the above differential equations (34) is chosen in preference to any other because for a solution $x_{1}(\tau), x_{2}(\tau), \cdots, x_{n}(\tau)$ of $(34)$ the following identity in $\tau$ holds:

$$
f\left[x_{1}(\tau), x_{\mathbf{2}}(\tau), \cdots, x_{n}(\tau)\right] \equiv \tau+\text { const. }
$$


For if the left hand member of (35) be differentiated with respect to $\tau$, making use of (34) it is found that the resulting derivative is unity. Whence (35) follows directly.

Let there be given an ordinary point $(B)$ with coördinates $\left(b_{1}, b_{2}, \cdots, b_{n}\right)$. In a sufficiently small neighborhood of $(B)$ the denominator of the quotient in (34) giving $r$ is not zero, and in such a neighborhood the right hand members of the differential equations (34) possess continuous partial derivatives with respect to each of the $x$ 's. Further these right hand members do not involve $\tau$ at all. It follows from the fundamental existence theorems of ordinary differential equations that there exists a positive constant $e$ so small that for any point $(A)=\left(a_{1}, a_{2}, \cdots, a_{n}\right)$ in the $e$ neighborhood of $(B)$ and for any constant $\tau_{0}$ whatsoever, and range of $r$ within $e$ of $\boldsymbol{r}_{0}$, there exist functions

$$
x_{i}=h_{i}\left(a_{1}, a_{2}, \cdots, a_{n}, \boldsymbol{\tau}_{0}, \boldsymbol{r}\right) \quad(i=1,2, \cdots, n)
$$

which give for constant values of $\left(a_{1}, a_{2}, \ldots, a_{n}, x_{0}\right)$ and for a variation of $r$, a solution of (34) that passes through $(A)$ when $r=\tau_{0}$, and is the only such solution of (34). Further, for the above prescribed domain of $\left(a_{1}, a_{2}, \cdots, a_{n}, \tau_{0}\right)$ the right hand members of $\left(35^{\prime}\right)$ possess continuous partial derivatives of at least the first order with respect to all of their arguments.

Because the right hand members of (34) do not involve $r$ it follows that two solutions of (34), for which the points $\left(a_{1}, a_{2}, \ldots, a_{n}\right)$ are the same but for which $\tau_{0}$ differs, will give the same set of points $\left(x_{1}, x_{2}, \cdots, x_{n}\right)$, assigned however by $\left(35^{\prime}\right)$ to different values of $\tau$. What is here desired is to obtain all of the orthogonal trajectories in a form which will be the simplest possible for our purposes. This end will be served by setting

Upon writing

$$
\tau_{0}=f\left(a_{1}, a_{2}, \cdots, a_{n}\right) \text {. }
$$

$h_{i}\left[a_{1}, a_{2}, \cdots, a_{n}, f\left(a_{1}, a_{2}, \cdots, a_{n}\right), \tau\right]=H_{i}\left(a_{1}, a_{2}, \cdots, a_{n}, \tau\right)(i=1,2, \cdots, n)$

$\left(35^{\prime}\right)$ reduces to

$$
x_{i}=H_{i}\left(a_{1}, a_{2}, \cdots, a_{n}, \tau\right)
$$

For initial conditions we now have

$$
a_{i}=H_{i}\left[a_{1}, a_{2}, \cdots, a_{n}, f\left(a_{1}, a_{2}, \cdots, a_{n}\right)\right] \text {, }
$$

an identity in all of the $a_{i}$ 's in the neighborhood of $(B)$. If the solution of (34), namely $x_{1}(\tau), x_{2}(\tau), \ldots, x_{n}(\tau)$, appearing in (35) be now understood 
to be a solution given by (36), a substitution in (35) of $\tau=f\left(a_{1}, a_{2}, \cdots, a_{n}\right)$ will show that the constant in (35) is zero. Thus for all solutions of (34) given by (36) there results the identity in $x$

$$
f\left[x_{1}(\tau), x_{z}(\tau), \cdots, x_{n}(\tau)\right] \equiv \tau \text {. }
$$

Thus we have proved the following lemma:

Lemma 5. The trajectories orthogonal to the manifolds $f=c$ can be so represented that the parameter $\tau$ at any one of their points equals the value of $f$ at that point.

7. Invariance of the connectivity numbers of the complex $f \leqq c$ with variation of $c$ through ordinary values of $f$. The following lemma will now be proved.

Lemma 6. Let $a$ and $b(a<b)$ be two ordinary values of $f$ in $S$ such that the interval betwcen $a$ and $l$ contains only ordinary values of $f$; then the set of points in $S$ satisfying

$$
f \leqq a
$$

can be put into one-to-one continuous correspondence with the set of points in $S$ satisfying

$$
f \leqq b
$$

The constant $a$ is by hypothesis not a critical value of $f$. Since there are altogether at most a finite number of critical values of $f$ there can be chosen a constant $\alpha$ less than $a$ and differing from $a$ by so little that between an $a$ and $a$ there is no constant equal to a critical value of $f$. Denote by $H$ a set of orthogonal trajectories in which there is just one trajectory passing through each point of

$$
f=\alpha \text {. }
$$

Let these trajectories be represented in the form described in Lemma 5 in which the value of the parameter $\tau$ at each point equals the value of $f$ at that point. 'The point on each trajectory that lies on (41) will correspond to the parameter value $\tau=\alpha$.

Since there is no critical value of $f$ equal to a constant between $\alpha$ and $b$ each trajectory that starts from a point of (41) can be continued through an arc corresponding to a range of values of $\boldsymbol{\tau}$ from $\alpha$ to $b$. On these arcs the parameter values $\tau=a$ and $\tau=b$, respectively, will correspond to points on $f=a$ and $f=b$. To the complete set of values of $\tau$ satisfying

$$
\alpha \leqq \tau \leqq b
$$


there will correspond points satisfying

$$
\iota \leqq f \leqq l
$$

Further, the set of trajectories $H$, including as it does a trajectory through each point of (41), if continued through the interval (42) will include at least one trajectory through each point $\left(x_{1}, x_{2}, \cdots, x_{n}\right)$ satisfying (43). For if

$$
\left(a_{1}, a_{2}, \cdots, a_{n}\right)=(A)
$$

be any point such that

$$
a \leqq f^{\prime}\left(a_{1}, a_{2}, \cdots, a_{n}\right) \leqq l
$$

a trajectory through $(A)$, represented as described in Lemma 5, will pass through $(A)$ for a parameter value $r=f\left(a_{1}, a_{2}, \cdots, a_{n}\right)$ and if continued in the sense of decreasing $r$ will reach a point on (41) when $r$ reaches the value $x=\alpha$. Finally, it follows from the fundamental existence theorem stated in $\$ 6$ that there is not more than one of these trajectories through each point satisfying (43).

The correspondence whose existence is affirmed in the lemma can now be set ul). First observe that the points satisfying (39) consist of the sum of the points satisfying

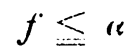

and those satisfying:

$$
a<f \leqslant a,
$$

while the points satisfying (40) consist of the points satisfying (45) together with the points satisfying

$$
\alpha<f \leqq b .
$$

Thus to put the points satisfying (39) into correspondence with the points satisfying (40) the points satisfying (45) are first made to correspond to themselves. The points satisfying (46) are then put into correspondence with those satisfying (47) by requiring that any point satisfying (46) that arises on the trajectory through it from a value of $r$ that divides the interval from $\alpha$ to $a$ in a certain ratio, shall correspond to that point satisfying (47) that lies on the same trajectory and arises from a value of $r$ that divides the interval from $\alpha$ to $b$ in the same ratio.

That the latter correspondence is one-to-one follows from the fact that the trajectories concerned pass through each of the points concerned with one and only one trajectory through each point. That this correspon- 
dence is continuous follows from the nature of the dependence of the points of the trajectories on $\tau$ and on their initial points on (41). Further, the correspondence of the points satisfying (45) with themselves obviously joins up in a continuous manner with the correspondence of the points satisfying (46) with those satisfying (47). Thus the lemma is proved.

\section{CRITICAL POINTS OF THE TYPE ZERO}

8. The connectivity numbers of the complex $f \leqq m+e^{\mathrm{s}}$ where $m$ is the absolute minimum of $f$. Let $e$ be a positive constant so small that between $m$ and $m+e^{2}$ there is no constant equal to a critical value of $f$. Under the boundary conditions $a, \S 4, f$ will take on its absolute minimum at one or more points all interior to $S$. Let $P$ be one such point. It follows from Lemma 4 of $\S 3$ that the points $\left(x_{1}, x_{2}, \cdots, x_{n}\right)=(X)$ neighboring $P$ can be transformed in a one-to-one continuous manner into the points $\left(y_{1}, y_{2}, \cdots, y_{n}\right)=\left(Y^{\prime}\right)$ neighboring the origin in such a manner that

$$
f\left(x_{1}, x_{2}, \cdots, x_{n}\right)-m=y_{1}^{2}+y_{2}^{2}+\cdots+y_{n}^{2} .
$$

Thus the points in the neighborhood of $P$ satisfying

$$
f\left(x_{1}, x_{2}, \cdots, x_{n}\right) \leqq m+e^{2},
$$

for a sufficiently small positive constant $e$, will correspond in a one-to-one continuous manner to the points satisfying

$$
y_{1}^{2}+y_{2}^{2}+\cdots+y_{n}^{2} \leqq e^{2}
$$

or what might be described as the points interior to and on an $(n-1)$ dimensional hypersphere in an $n$-dimensional space. Such a set of points would constitute, in the terms of analysis situs, an $n$-cell and its boundary. With this understood we can state the following lemma.

Lemma 7. If $f$ talies on its absolute minimum $m$ in $S$ at $s$ distinct points of $S$, then for a sufficiently small positive constant e the set of points in $S$ satisfying

$$
f\left(x_{1}, x_{2}, \cdots, x_{n}\right) \leqq m+e^{2}
$$

constitutes $s$ distinct $n$-cells and their boundaries.

From this lemma and the definitions of the connectivity numbers* we have the following theorem.

* V, loc. cit. 
Theorem 1. If $f$ takes on its absolute minimum $m$ in $S$ at $s$ distinct points of $S$ then for a sufficiently small positive constant e the set of points in $S$ satisfying

$$
f\left(x_{1}, x_{2}, \cdots, x_{n}\right) \leqq m+e^{2}
$$

makes up an $n$-dimensional complex with the connectivity numbers $R_{0}=s$, $R_{1}=R_{2}=\ldots=R_{n}=1$.

9. A critical point of type zero at which $f$ is not equal to its absolute minimum.

Theorem 2. If a and $b(a<b)$ are any two ordinary values of $f$ in $S$ between which there is just one critical value of $f$, say $c$, taken on at $p_{0}$ critical points all of the zeroth type, then the connectivity number's $R_{i}$, of the set of points in $S$ satisfying

$$
f\left(x_{1}, x_{2}, \cdots, x_{n}\right) \leqq b,
$$

differ from the connectivity numbers $R_{1}^{\prime}$ of the set of points in $S$ satisfying

only in that

$$
f\left(x_{1}, x_{2}, \cdots, x_{n}\right) \leqq a
$$

$$
R_{0}=R_{0}^{\prime}+p_{0}
$$

Let $P$ be any one of the $p_{0}$ critical points of the zeroth type. If $e$ be a sufficiently small positive constant it follows from I-emma $4, \S 3$, that the points $\left(x_{1}, x_{z}, \cdots, x_{n}\right)$ neighboring $P$ and satisfying

$$
f \leqq c+e^{2}
$$

can be made to correspond in a one-to-one continuous manner to a set of points $\left(y_{1}, y_{2}, \cdots, y_{n}\right)$ satisfying

$$
y_{1}^{2}+y_{2}^{2}+\cdots+y_{n}^{2} \leq e^{2} .
$$

Now the points satisfying (51) make up an $n$-cell and its boundary. There will thus be associated with each of the $p_{0}$ critical points an $n$-cell and its boundary consisting of points satisfying (50). For $e$ sufficiently small these $n$-cells will be distinct from each other and from the set of remaining points, say $C_{n}$, satisfying (50).

The set $C_{n}$ is bounded by points at which $f=c+e^{2}$, and contains only those critical points which are already contained among the points satisfying (49). With the aid of the orthogonal trajectories as used in the proof of Lemma 6 , it is easy to show that $C_{n}$ can be put into one-to-one continuous 
correspondence with the set of points satisfying (49). The connectivity numbers of $C_{n}$ are accordingly equal to the connectivity numbers $R_{i}^{\prime}$ of the complex (49).

If now the $p_{0} n$-cells and their boundaries be added to the complex $C_{n}$ there will result the entire set of points satisfying (50) and the only connectivity number to be thereby changed will be $R_{0}^{\prime}$, which will be increased by $p_{0}$. If finally we suppose $e$ originally chosen so small that $c+e^{2}<b$, it follows from Lemma 6 that the set of points satisfying (50) can be put into one-to-one correspondence with the set of points satisfying (48). Hence (48) defines a complex with the same connectivity numbers as the complex defined by (50). Retracing the steps it is seen that the relation between the connectivity numbers of the complexes (48) and (49) is as stated.

CRitical Polvts of TyPES $1,2, \cdots, n-1$

10. Outline of the method to be followed. Throughout this chapter we shall assume that we are dealing with a critical value $c$ taken on by $f$ at just one critical point of the $k$ th type, where $k$ may be any one of the integers $1,2, \cdots, n-1$. We can and will suppose $e$ to be so small a positive constant that there is no critical value of $f$ between $c-e^{2}$ and $c+e^{2}$ or equal to $c-e^{2}$ or $c+e^{2}$. It is the aim of this chapter to determine the difference between the connectivity numbers of the complex of points $(X)$ satisfying

$$
f \leqq c-e^{2}
$$

and the complex of points $(X)$ satisfying

$$
f \leqq c+e^{z}
$$

To accomplish this we will first show that the complex (52) can be put into one-to-one continuous correspondence with the complex (53) provided we exclude a properly chosen neighborhood of the given critical point. This will be accomplished with the aid of the orthogonal trajectories already used in $\S 7$. The problem then will be resolved into one of determining the difference between the connectivities of the complex (53) and of the complex (53) with this neighborhood excluded.

11. Simultaneous reduction of $f\left(x_{1}, x_{2}, \ldots, x_{n}\right)$ and the differential equations of the orthogonal trajectories to a simple form. It follows from Lemma $2, \S 2$, that, in the neighborhood of a critical point $\left(x_{1}^{0}, x_{2}^{0}, \cdots, x_{n}^{0}\right)$ at which $f=c$, 


$$
f-c=\sum_{i j} b_{i j}\left(x_{i}-x_{i}^{0}\right)\left(x_{j}-x_{j}^{0}\right) \quad(i, j=1,2, \cdots, n),
$$

where each $b_{i j}$ is a continuous function of the $x$ 's possessing continuous first partial derivatives, and where we can suppose that $b_{i j}=b_{j i}$. In the following the matrix whose element in the $i$ th row and $j$ th column is given by a symbol such as $b_{i j}$ will itself be denoted be the same symbol $b$ without the subscripts. Conjugates of matrices will be indicated by adding a prime.

LEMmA A. In the neighborhood of a critical point $\left(x_{1}^{0}, x_{2}^{0}, \ldots, x_{n}^{0}\right)=\left(X_{0}\right)$ there exists a real transformation of the form

$$
x_{i}-x_{i}^{0}=\sum_{j} a_{i j} z_{j} \quad(i, j=1,2, \ldots, n)
$$

such that (54) becomes

$$
\sum_{i j} b_{i j}\left(x_{i}-x_{i}^{0}\right)\left(x_{j}-x_{j}^{0}\right)=r_{1} z_{1}^{2}+\cdots+r_{n} z_{n}^{2},
$$

where $r_{1}, r_{2}, \cdots, r_{k}$ are negative constants and $r_{k+1}, r_{k+2}, \cdots, r_{n}$ are positive constants, while in (55) each $a_{i j}$ is a function of the z's possessing continuous first partial derivatives in the neighborhood of the origin of the space of the $z$ 's, and at the origin the matrix equation

$$
a(0,0, \cdots, 0) a^{\prime}(0,0, \ldots, 0)=\mathrm{I}
$$

holds where I is the unit matrix.

Proof. From the results of $\S 3$ it follows that in the neighborhood of the given critical point there exists a transformation

under which

$$
x_{i}-x_{i}^{0}=\sum_{j} e_{i j} y_{j} \quad(i, j=1,2, \cdots, n)
$$

$$
\sum_{i j} b_{i j}\left(x_{i}-x_{i}^{0}\right)\left(x_{j}-x_{j}^{0}\right)=-y_{1}^{2}-y_{2}^{2}-\cdots-y_{k}^{2}+y_{k+1}^{2}+y_{k+2}^{2}+\cdots+y_{n}^{2}
$$

where each $e_{i j}$ is a function of the $y$ 's possessing continuous first partial derivatives in the neighborhood of the origin of the space of the $y$ 's. Denote by $\eta_{i j}$ the value of $e_{i j}$ at the origin of the $y$ 's. Suppose that under the transformation

$$
\begin{aligned}
x_{i} & =\sum_{j} \eta_{i j} y_{j} \quad(i, j=1,2, \cdots, n), \\
\sum_{i} x_{i}^{2} & =\sum_{i j} \gamma_{i j} y_{i} y_{j} .
\end{aligned}
$$


According to the theory of pairs of quadratic forms the quadratic forms in the right hand members of (59) and (61), by a real non-singular transformation with constant coefficients of the form

$$
y_{i}=\sum_{j} \delta_{i j} z_{j} \quad(i, j=1,2, \cdots, n)
$$

can be reduced to a pair of forms

$$
\begin{gathered}
r_{1} z_{1}^{2}+r_{2} z_{2}^{2}+\cdots+r_{n} z_{n}^{2} \\
z_{1}^{2}+z_{2}^{2}+\cdots+z_{n}^{2} .
\end{gathered}
$$

The transformation obtained by expressing the $x$ 's in (58) in terms of the $z$ 's in (62) is the one whose existence is affirmed in the lemma. For the matrix of this combined transformation, namely $e \delta$, reduces at the origin to $\eta \delta$, and $\eta \delta$ is the matrix of a transformation under which

$$
\sum_{i=1}^{n} x_{i}^{2}=\sum_{i=1}^{n} z_{i}^{2}
$$

so that $\eta \delta$ is orthogonal as (57) requires. The remaining assertions of Lemma A follow directly.

Lемма B. In the neighborhood of the critical point $\left(X_{0}\right)$ there exists a real transformation

such that

$$
x_{i}-x_{i}^{0}=\sum_{j} g_{i j} y_{j} \quad(i, j=1,2, \cdots, n)
$$

$$
\sum_{i j} b_{i j}\left(x_{i}-x_{i}^{0}\right)\left(x_{j}-x_{j}^{0}\right)=-y_{1}^{2}-y_{2}^{2}-\cdots-y_{k}^{2}+y_{k+1}^{2}+\cdots+y_{n}^{2},
$$

while the differential equations of the orthogonal trajectories to the manifolds $f=$ const. if taken in the form

$$
\frac{d x_{h}}{d t}=f_{h} \quad(h=1,2, \cdots, n)
$$

are transformed into the equations

$$
\frac{d y_{h}}{d t}=2 r_{h} y_{h}+\sum_{i j} A_{h i j} y_{i} y_{j}(h, i, j=1,2, \ldots, n)
$$

where $r_{1}, r_{2}, \cdots, r_{k}$ are positive constants and $r_{k+1}, r_{k+2}, \cdots, r_{n}$ are negative constants, where each $g_{i j}$ and $A_{h i j}$ is a continuous finction of the $y$ 's 
in the neighborhood of the origin and the $g_{i j}$ there possess continuous first partial derivatives.

Proof. With the aid of the representation of $f$ given in (54), (65) becomes

$$
\frac{d x_{h}}{d t}=2 \sum_{i} b_{h i}\left(x_{i}-x_{i}^{0}\right)+\sum_{i j} \frac{\partial b_{i j}}{\partial x_{h}}\left(x_{i}-x_{i}^{0}\right)\left(x_{j}-x_{j}^{0}\right) .
$$

It follows from (C), $\S 2$, that each $b_{h i}$ equals its value, say $\beta_{h i}$ at $\left(X_{0}\right)$, plus a sum of terms each a product of a difference $\left(x_{j}-x_{j}^{0}\right)$ multiplied by a function of the $x$ 's continuous in the neighborhood of $\left(X_{0}\right)$. Thus (67) becomes

$$
\frac{d x_{h}}{d t}=2 \sum_{i} \beta_{h i}\left(x_{i}-x_{i}^{0}\right)+\sum_{i j} B_{h i j}\left(x_{i}-x_{i}^{0}\right)\left(x_{j}-x_{j}^{0}\right)
$$

where each $B_{h i j}$ is a continuous function of the $x$ 's in the neighborhood of the origin. The transformation (55) of Lemma A will be applied to (68). Remembering that the value of $a_{i j}$ at the origin is written as $\alpha_{i j}$, it is seen that (55) may be written

$$
x_{h}-x_{h}^{0}=\sum_{i} \alpha_{h i} z_{i}+\sum_{i j} C_{h i j} z_{i} z_{j}
$$

where each $C_{h i j}$ is a continuous function of the $z$ 's in the neighborhood of their origin.

The transformation (55) applied to (68) gives

$$
\frac{d z_{h}}{d t}=2 \sum_{i} p_{h i} z_{i}+\sum_{i j} D_{h i j} z_{i} z_{j}
$$

where the elements $p_{h i}$ are constants such that, in matrix notation,

$$
p=\alpha^{-1} \beta \alpha
$$

and each $D_{h i j}$ is a continuous function of the $z$ 's in the neighborhood of their origin. But according to (57) $\alpha \alpha^{\prime}=1$ so that $\alpha^{\prime}=\alpha^{-1}$. Hence (71) may be written as

$$
p=\alpha^{\prime} \beta \alpha .
$$

But from (55) and (56) it follows that

$$
a^{\prime} b a=r .
$$

Matrix relation (73) becomes at the origin

$$
\boldsymbol{\alpha}^{\prime} \beta \boldsymbol{\alpha}=\boldsymbol{r}
$$


so that from (74) and (72) we have

Thus (70) takes the form

$$
p=r
$$

$$
\frac{d z_{h}}{d t}=2 r_{h} z_{h}+\sum_{i j} D_{h i j} z_{i} z_{j}
$$

Finally the transformation

$$
y_{h}=\sqrt{\left|r_{h}\right|} z_{h} \quad(h=1,2, \ldots, n)
$$

changes the right hand member of (56) to the right hand member of (64) and takes (75) into the form (66). Thus the transformation obtained by combining (55) and (76) so as to eliminate the $z$ 's is of the nature required of (63) and the lemma is proved.

12. The correspondence between the complex $f \leqq c-e^{2}$ and the complex $f \leqq c+e^{2}$ with the points (79) excluded. We are concerned with a critical value $c$ taken on by $f$ at just one critical point $\left(X_{0}\right)$ of the $k$ th type $(0<k<n)$. Let the points $(X)$ in the neighborhood of $\left(X_{0}\right)$ be subjected to the transformation $(63)$ of Lemma $B, \S 11$, so that in terms of the new variables $\left(y_{1}, y_{\Sigma}, \cdots, y_{n}\right)=(Y)$

where

$$
f-c=q^{2}-p^{2}
$$

$$
\begin{aligned}
& p^{2}=y_{1}^{2}+y_{2}^{2}+\cdots+y_{k}^{2}, \\
& q^{2}=y_{k+1}^{2}+\cdots+y_{n}^{2} .
\end{aligned}
$$

It is the purpose of this section to show that for a proper choice of constants $a$ and $e(a>e>0)$, the complex of points satisfying $f \leqq c-e^{2}$ can be put into one-to-one continuous correspondence with the complex of points satisfying $f \leqq c+e^{2}$ provided we exclude from the latter complex those points which satisfy

$$
c-e^{2}<f \leqq c+e^{2}, \quad p^{2}<a^{2}
$$

The existence of this correspondence is affirmed in slightly different terms in the following lemma.

Lемма 8. If $c$ be a critical value of $f$ taken on by $f$ at just one critical point $\left(X_{0}\right)$, and if $\left(X_{0}\right)$ is of the type for which $0<k<n$, then for a proper choice of $a$ and $e, a>e>0$, the points $(X)$ satisfying

$$
f \leqq c-e^{2}
$$


can be put into one-to-one continuous correspondence with the set of points consisting of the points (80) together with the points satisfying

$$
c-e^{2}<f \leqq c+e^{2}, \quad p^{2} \geqq a^{2} .^{*}
$$

The latter inequality $p^{2} \geqq a^{2}$ has a meaning and is intended to have a meaning only where the variables $y_{1}, y_{2}, \cdots, y_{n}$ are defined (Lemma B), that is, in the neighborhood of the given critical point. To prove this lemma, Lemmas C, D, E, F, and G will first be established. For this purpose the boundary points of (81) will be grouped into the following sets of points: the points on $f=c+e^{2}$ satisfying

$$
f=c+e^{2}, \quad p^{2}>a^{2},^{*}
$$

the points on $f=c-e^{2}$ satisfying

$$
f=c-e^{2}, \quad p^{2}>a^{2},^{*}
$$

the points on $p^{2}=a^{2}$ satisfying

$$
p^{2}=a^{2}, \quad c-e^{2}<f \leqq c+e^{2},
$$

and the points

$$
p^{2}=a^{2}, \quad f=c-e^{2} .
$$

Lemma C. There exists a positive constant $d$ so small that when $a$ is chosen less than $d$, e can then be chosen so small that none of the trajectories orthogonal to the manifolds $f=$ const. passes through more than one point of (84) or its boundary.

Proof of Lemma C. For $e$ sufficiently small each point of (84) lies arbitrarily near some point satisfying

$$
p^{2}=q^{2}=a^{2}
$$

Let $(B)=\left(b_{1}, b_{2}, \cdots, b_{n}\right)$ be a point satisfying (86). Let $(U)=\left(u_{1}, u_{2}, \cdots, u_{n}\right)$ be a point in the space of the $y$ 's near $(B)$. The solutions of $(66)$ which pass through $(U)$ when $t=0$ are representable for $t$ near 0 in the form

for which

$$
y_{h}=Y_{h}\left(u_{1}, u_{2}, \cdots, u_{n}, t\right) \quad(h=1,2, \ldots, n),
$$

$$
u_{h} \equiv Y_{h}\left(u_{1}, u_{2}, \cdots, u_{n}, 0\right)
$$

* Where the latter condition has no meaning through lack of definition of $p$ it will be supposed satisfied vacuously. 
where each $Y_{h}$ possesses continuous partial derivatives with respect to all of its arguments for $(U)$ neighboring $(B)$ and $t$ neighboring 0 .

If now any point $(U)$ neighboring $(B)$ be given, the trajectory passing through $(U)$ for $t=0$ meets the manifold $p^{2}=a^{2}$ in as many points as there are solutions $\left(y_{1}, y_{2}, \ldots, y_{n}, t\right)$ of the equations

$$
\begin{aligned}
& y_{h}=Y_{h}\left(u_{1}, u_{2}, \cdots, u_{n}, t\right), \\
& p^{2}=a^{2} .
\end{aligned}
$$

Equations (89) will be written in the form

$$
\begin{aligned}
& y_{h}-Y_{h}\left(u_{1}, u_{2}, \ldots, u_{n}, t\right)=0 \\
& y_{1}^{2}+y_{2}^{2}+\cdots+y_{k}^{2}-a^{2}=0 .
\end{aligned}
$$

The jacobian $J$ of the left hand members of (90) with respect to $y_{1}, y_{2}, \ldots, y_{n}$ and $t$ for $t=0$ is obtained by bordering an $n$-square unit matrix $\mathrm{I}$ as follows (Lemma B, § 11):

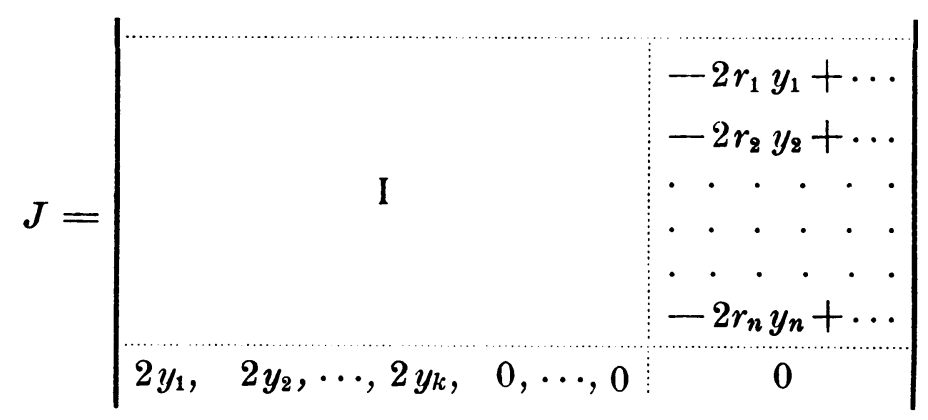

where the terms omitted in the last column are of the form $A_{i j} y_{i} y_{j}$, where each $A_{i j}$ is a continuous function of the $y$ 's in the neighborhood of the origin. The value of this jacobian when $(U)=(Y)=\left(b_{1}, b_{2}, \ldots, b_{n}\right)$ is

$$
J=4 \sum_{h} r_{h} b_{h}^{2}+\sum_{m i j} B_{m i j} b_{m} b_{i} b_{j} \quad\left(\begin{array}{r}
m, i, j=1,2, \ldots, n ; \\
h=1,2, \ldots, k
\end{array}\right)
$$

where each $B_{m i j}$ is a continuous function of the $b$ 's in the neighborhood of the origin.

Recalling that $r_{1}, r_{y}, \ldots, r_{k}$ are all negative constants, let $m$ and $M$ be two positive constants such that

Then

$$
m<-4 r_{h}<M \quad(h=1,2, \ldots, k) .
$$

$$
m \sum b_{h}^{2}<-4 \sum r_{h} b_{h}^{2}<M \sum b_{h}^{2} .
$$


Since the point $(B)$ satisfies (86),

Hence from (92)

$$
a^{2}=\sum_{h} b_{h}^{2}
$$

$$
m a^{2}<-4 \sum_{h} r_{h} b_{h}^{2}<M a^{2} \quad(h=1,2, \cdots, k)
$$

That is

$$
4 \sum_{h} r_{h} b_{h}^{2}=R a^{2}
$$

where $R$ is a function of $\left(b_{1}, b_{2}, \ldots, b_{n}\right)$ such that

$$
m<|R|<M \text {. }
$$

Now the point $(B)$ satisfies $p^{2}=q^{2}=a^{2}$, from which it follows that

$$
\left|b_{i}\right| \leqq a
$$

Moreover since the functions $B_{m i j}$ in (91) are continuous functions of the point $(B)$ for $(B)$ in the neighborhood of the origin, there exists a constant $H$ such that for that neighborhood

$$
\left|B_{m i j}\right|<H \text {. }
$$

For this neighborhood it follows from (95) and (96) that

$$
\sum_{m i j} B_{m i j} b_{m} b_{i} b_{j}<n^{3} H a^{3}
$$

where $n^{3}$ appears since there are not more than $n^{3}$ terms $b_{m} b_{i} b_{i}$. Thus

$$
\sum_{m i j} B_{m i j} b_{m} b_{i} b_{j}=T a^{3}
$$

where $T$ is a function of the point $(B)$ such that in the neighborhood of the origin

$$
|T|<n^{3} H
$$

From (91), (93), and (97) it follows that

$$
J=R a^{2}+T a^{3}
$$

We can and do hereby choose a positive constant $d$ so small that for $0<a<d$

$$
J \neq 0 \text {. }
$$


For this choice of $a$ none of the segments of the trajectories with points all lying sufficiently near $(B)$ pass through more than one point of $p^{2}=a^{2}$ neighboring $(B)$. To show that none of these trajectories return to points of (84) it will be useful to take again that representation of the trajectories in which the parameter $\boldsymbol{r}$ at any point on a trajectory equals the value of $f$ at that point. For $e$ sufficiently small the points on the trajectories passing through some point in a sufficiently small neighborhood of $(B)$ and for which

$$
c-e^{2} \leqq \tau \leqq c+e^{2}
$$

will all lie arbitrarily near $(B)$ and hence have at most one intersection with $p^{2}=a^{2}$. But these trajectories no matter how extended will not return to points of (84) or its boundary, for points on the extended trajectories will be points for which

$$
\tau>c+e^{\mathrm{a}} \quad \text { or } \quad \tau<c-e^{\mathbf{2}}
$$

and hence will be points at which

$$
f>c+e^{2} \text { or } f<c-e^{2},
$$

and hence will not be points satisfying (84) or its boundary.

The following lemma follows from the above proof, and in particular from the non-vanishing of the above jacobian.

LEMna D. For a choice of a such that the above jacobian is not zero and for any point $(U)$ sufficiently near some point of

$$
p^{2}=q^{2}=a^{2},
$$

the trajectory passing through $(U)$ for $t=0$ meets the manifold

$$
p^{2}=a^{2}
$$

in a point $(Y)$ whose coördinates and parameter value $t$ are continuous functions of the coördinates of $(U)$ and are provided with continuous first partial derivatives.

The proof of the following lemma could be given along the same lines as the proof of Lemmas C and D.

Lemma E. For a sufficiently small positive constant a, and corresponding to this a, a sufficiently small positive constant $e$, and for any point $(U)$ sufficiently near some point of

$$
p^{2}=q^{2}=a^{2},
$$


the trajectory which passes through $(U)$ when $t=0$ meets the manifold

$$
p^{2}-q^{2}=e^{2}
$$

in a point $(Y)$ whose coördinates and parameter value $t$ are continuous functions of the coördinates of $(U)$ and are provided with continuous first partial derivatives.

The following lemma will now be proved:

Leмma F. For a sufficiently small positive constant a, and corresponding to this a, a sufficiently small positive constant e, all of the trajectories passing through points of (84) pass through points of (83).

The equations (84) may be written in terms of the point $(Y)$ as

or again as

$$
-e^{2} \leqq p^{2}-q^{2}<e^{2}, \quad p^{2}=a^{2},
$$

$$
p^{2}=a^{2}, \quad a^{2}-c^{2}<q^{2} \leqq a^{2}+e^{2},
$$

while (83) may be written as

$$
p^{2}-q^{2}=e^{2}, \quad p^{2}>a^{2} .
$$

To distinguish between a point $(Y)$ on (99) and a point $(Y)$ on (100), any point $(Y)$ on $(99)$ will be written as $(Y)=(U)=\left(u_{1}, u_{2}, \cdots, u_{n}\right)$. Now (99) and (100) have as common boundary points the points satisfying

$$
p^{2}=a^{2}, \quad q^{2}=a^{2}-e^{2}
$$

Let $(B)=\left(b_{1}, b_{2}, \cdots, b_{n}\right)$ be any point on (101). The complete set of points on (99) consists of the points on the following family of straight lines:

$$
\begin{aligned}
& u_{1}=b_{1}, \\
& u_{2}=b_{2}, \\
& \cdot \cdot \cdot \cdot \cdot, \\
& u_{k}=b_{k}, \\
& u_{k+1}=m b_{k+1}, \\
& \cdot \cdot \cdot \cdot \cdot \cdot, \\
& u_{n}=m b_{n},
\end{aligned}
$$

where $m$ is a parameter varying on each straight line on the interval

$$
1<m \leqq \sqrt{\frac{a^{2}+e^{2}}{a^{2}-e^{2}}} .
$$


For $m=1,(U)=(B)$. If $m$, considered ais the single independent variable, vary on its interval (103), $(U)$ will then vary on the straight line (102), and the trajectory through $(U)$, according to Lemma $\mathrm{E}$, will meet $p^{2}-q^{2}=e^{2}$ in a point $(Y)$ whose coördinates and parameter value $t$ will be continuous functions of $m$ provided with continuous derivatives with respect to $m$. $(U),(Y)$, and $t$, thus depending upon $m$, satisfy (87) making (87) an indentity in $\mathrm{m}$. The lemma will follow readily if it can be shown that as $m$ increases on its interval, and $(Y)$ depends on $m$ as just described, $p^{2}=y_{1}^{2}+y_{2}^{2}+\cdots+y_{k}^{2}$, considered as a function of $m$, increases.

To this end let (87) be differentiated with respect to $m$. At $m=1$ and hence $t=0$ (cf. (66)),

$$
\begin{aligned}
& \left.\frac{d y_{h}}{d m}=\quad\left[2 r_{h} y_{h}+\sum_{i j} A_{h i j} y_{i} y_{j}\right] \frac{d t}{d m} \quad \begin{array}{c}
h=1,2, \ldots, k \\
i, j=1,2, \ldots, n
\end{array}\right), \\
& \frac{d y_{h}}{d m}=b_{h}+\left[2 r_{h} y_{h}+\sum_{i j} A_{h i j} y_{i} y_{j}\right] \frac{d t}{d m} \quad(h=k+1, \ldots, n) .
\end{aligned}
$$

If the two members of the equation in (104) which gives $d y_{n} / d m$ be multiplied by $2 y_{h}(h=1,2, \ldots, k)$ and the resulting $k$ equations added, the left hand sum will become $d p^{2} / d n$, and if in the right hand sum we set $(Y)=(U)=(B)$ we will have

$$
\frac{d p^{2}}{d m}=4\left[\sum_{h} r_{h} b_{h}^{2}+\cdots\right] \frac{d t}{d m} \quad(h=1,2, \ldots, k),
$$

where the terms omitted here and in the subsequent equations of this proof are sums of terms of the form

$$
B_{h i j} b_{h} b_{i} b_{j},
$$

where $B_{h i j}$ is a continuous function of all of the $b$ 's in the neighborhood of the origin.

Similarly, upon multiplying the two members of the equation in (105) that gives $d y_{h} / d m$ by $2 y_{h}(h=k+1, \ldots, n)$ and adding the resulting $n-k$ equations we will have at $(Y)=(U)=(B)$, using (101),

$$
\frac{d q^{2}}{d m}=2\left(a^{2}-e^{2}\right)+4\left[\sum_{h} r_{h} b_{h}^{2}+\cdots\right] \frac{d t}{d m} \quad(h=k+1, \cdots, n) .
$$

Now the coördinates of $(Y)$ considered as the above functions of $m$ satisfy $p^{2}-q^{2}=e^{2}$ identically. Thus

$$
\frac{d q^{2}}{d m}=\frac{d p^{2}}{d m} .
$$


Upon eliminating $\frac{d q^{2}}{d m}$ and $\frac{d t}{d m}$ from (106), (107), and (108), we have

$$
\begin{aligned}
\frac{d p^{2}}{d m} & =\frac{2\left[\sum_{h} r_{h} b_{h}^{2}+\cdots\right]\left(a^{2}-e^{2}\right)}{\sum_{h} r_{h} b_{h}^{2}-\sum_{i} r_{i} b_{i}^{2}+\cdots} \quad\left(\begin{array}{l}
h=1,2, \ldots, k ; \\
i=k+1, \ldots, n
\end{array}\right) \\
& =\frac{2\left[\sum_{h} r_{h} b_{h}^{2}+\cdots\right]\left(a^{2}-e^{2}\right)}{-\sum_{j}\left|r_{j}\right| b_{j}^{2}+\cdots} \quad(j=1,2, \ldots, n) .
\end{aligned}
$$

Now if $a$ be a sufficiently small positive constant and $e$ be less, say, than $a / 2$, it can be shown by the methods used at the end of the proof of Lemma $C$ that the expression (109) has the sign of

and hence is positive.

$$
\frac{2 \sum_{h} r_{h} b_{h}^{2}}{-\sum_{j}\left|r_{j}\right| b_{j}^{2}},
$$

For this choice of $a$ and $e, d p^{2} / d m$ is then positive at $(B)$, that is, when $m=1$. For $a$ fixed $(a>0)$ and for $e$ sufficiently small, the interval (103) will be arbitrarily small. Hence for $e$ sufficiently small $d p^{2} / d m$ will be positive throughout the interval (103). But for $m=1, p^{2}=a^{z}$. Thus on the interval (103) $p^{2}>a^{2}$, and the lemma follows directly.

The preceding proof requires only a formal alteration to serve as the proof of the following lemma.

Lemma G. For a sufficiently small positive constant a and, corresponding to this a, a sufficiently small positive constant $c$, none of the trajectories passing through points of (84) or its boundary will pass through a point of (82).

Proof of Lemma 8. Let us return to that form of representation of the orthogonal trajectories in which the parameter $\tau$ at any point on a trajectory equals the value of $f$ at that point. Let $(X)$ be any interior point of (81). On the trajectory through $(X)$ there will be a first point $P$ preceding $(X)$ in the sense of a decreasing $\tau$ which is on the boundary of (81), and a first point $Q$ following $(X)$ in the sense of an increasing $\tau$ which is on the boundary of (81). It follows from Lemmas $\mathrm{C}$ and $\mathrm{G}$ that neither $P$ nor $Q$ can lie on (85). $P$ will lie then either on (83) or else on (84), while $Q$ will lie on (82) or else on (84). There are four possibilities:
(a) $P$ on (84),
$Q$ on $(84)$,
(b) $P$ on (84), $Q$ on (82), 
(c) $P$ on (83), $Q$ on (84),

(d) $P$ on (83), $Q$ on (82).

The combination (a) is impossible according to Lemma $C$, while combination (b) is impossible according to Lemma G.

Thus $P$ always lies on (83). Further, the points $P$ will include each point $P_{1}$ on (83). For there are no boundary points of (81) other than points $f=c-e^{2}$ neighboring $P_{1}$. Hence all of the points neighboring $P_{1}$ for which $f>c-e^{2}$ will be interior points of (81). Hence on a trajectory through $P_{1}$ the points neighboring $P_{1}$ for which $\boldsymbol{r}>c-e^{2}$ will be interior points of (81). Thus $P_{1}$ will be one of the points $P$.

According to (c) and (d) all of the points $Q$ lie either on (82) or else on (84). Further, the points $Q$ include all of the points of (82), a fact which can be proved after the manner of proof in the preceding paragraph. The points $Q$ also include all the points $Q_{1}$ of (84). For according to Lemma $F$ a trajectory which passes through a point $Q_{1}$ on (84) in the sense of a decreasing $\tau$ will meet (83) in a point, say $P_{1}$. Now any point of (83) such as $P_{1}$ has just been proved to be a point $P$. Since there can be no point on (82) or (84) between $P_{1}$ and $Q_{1}, Q_{1}$ must be a point $Q$. Thus the points $Q$ include all of the points of (82) and (84). To sum up, the segments $P Q$ include all of the points of (81) and the boundary of (81) save the points of (85). At each of the points of (85) it is convenient to suppose a point $P$ coincident with a point $Q$.

Let $\tau_{1}$ and $\tau_{2}$ be respectively the values of $\tau$ at a point $P$ and corresponding $Q$. The value of $\tau_{1}$ is $c-e^{2}$. The position of $Q$ and value of $\tau_{2}$ are readily seen to be continuous functions of the position of $P$. To define the correspondence whose existence is affirmed in Lemma 8 let each trajectory $P Q$ be continued from $P$ in the sense of a decreasing $c$ to a point $R$ at which $\tau=\tau_{0}$, where

$$
\tau_{1}-\tau_{0}=\tau_{2}-\tau_{1} .
$$

The new trajectory segments $R P$ will consist of points satisfying (80). Now let every point of (80) not on a segment $R P$ correspond to itself, and let every point of (80) with parameter value $\tau$ on a segment $R P$ correspond to that point on $R P Q$ with parameter value $\boldsymbol{t}^{\prime}$, at which

$$
\left(\tau^{\prime}-\tau_{0}\right)=2\left(\tau-\tau_{0}\right) .
$$

The correspondence thereby established is readily seen to be of the nature required in the lemma to be proved.

13. Incidence relations between the boundary of $D_{n}$ and the remainder of the complex $f \leqq c+e^{2}$. Following the general outline 
of the method given in $\S 10$ this section is devoted first to proving that the set of points $D_{n}$ satisfying

$$
-e^{2} \leqq q^{2}-p^{2} \leqq e^{2}, \quad p^{2} \leqq a^{2}, \quad 0<. e<a,
$$

make up an $n$-cell $a_{n}$ and its boundary, and secondly to investigating the incidence relations between $D_{n}$ and the remainder of the complex of points satisfying $f \leqq c+e^{2}$.

For the moment let $p$ and $q$ in (110) be interpreted as the rectangular cartesian coördinates $(p q)$ of a point in a $p, q$ plane. So interpreted the points $(p q)$ which satisfy (110) consist of all those points lying between the two conjugate hyperbolas

$$
p^{2}-q^{2}=e^{2}, \quad q^{2}-p^{2}=e^{2}
$$

which also lie between the two straight lines

$$
p=a, \quad p=-a
$$

Since $a>e$ these two straight lines meet the two conjugate hyperbolas altogether in eight real points. The two-dimensional set of points in the $(p q)$ plane satisfying $(110)$ will be denoted by $D_{2}$. $D_{z}$ contains the origin and is symmetrical with respect to it.

We will now consider a transformation $T$ of the coördinates $(p q)$ into rectangular coördinates $(u v)$ which carries $D_{2}$ into a rectangle $E_{2}$ with vertices at the intersections of the straight lines $p= \pm a$ with the hyperbola $q^{2}-p^{2}=e^{2}$. By the methods of elementary analytic geometry it is seen that a straight line segment issuing from the origin in the $(p q)$ plane in any direction whatsoever meets the boundary of $D_{2}$ in just one point whose distance from the origin varies continuously with the angle the given line segment makes with the $p$ axis. The same is true of the boundary of the rectangle $E_{2} . \quad D_{2}$ and $E_{2}$ can be put into a one-to-one continuous correspondence $T$ by requiring that a point $(p q)$ on $D_{2}$ that divides the straight line segment from the origin to the boundary of $D_{\mathbf{z}}$ in a particular ratio shall correspond on $E_{2}$ to a point on the same straight line and so placed as to divide the line segment from the origin to the boundary of $E_{2}$ in the same ratio.

The following properties of the transformation $T$ will be used.

(A) Under the transformation $T$ the point $(p q)$ is related to its correspondent $(u v)$ as follows:

$$
\begin{array}{ll}
u=r\left(p^{2} q^{2}\right) p, & \\
v=r\left(p^{2} q^{2}\right) q, & r(00)=0,
\end{array}
$$


where $r\left(p^{2} q^{2}\right)$ is a positive, single-valued, continuous function of $p^{2}$ and $q^{2}$ for each pair $(p q)$ in $D_{2}$ not equal to $(00)$. The pair $(p q)$ is obviously also related to its correspondent $(u v)$ as follows:

$$
\begin{array}{ll}
p=\sigma\left(u^{2} v^{2}\right) u, & \\
q=\sigma\left(u^{2} v^{2}\right) v, & \sigma(00)=0
\end{array}
$$

where $\sigma\left(u^{2} v^{2}\right)$ is a positive, single-valued, continuous function of $u^{2}$ and $v^{2}$ for each pair $(u v)$ in $E_{2}$ not equal to (00).

(B) Under $T$ the points $(p q)$ on $D_{2}$, that is the points satisfying

$$
-e^{2} \leqq q^{2}-p^{2} \leqq e^{2}, \quad p^{2} \leqq a^{2}
$$

correspond to the points (uv) on $E_{2}$, that is the points satisfying

$$
u^{2} \leqq a^{2}, \quad v^{2} \leqq b^{2},
$$

where we have set

$$
a^{2}+e^{2}=b^{2}
$$

(C) The points on the boundary of $D_{2}$ satisfying

$$
p^{2}-q^{2}=e^{2}, \quad q^{2} \leqq a^{2}-e^{2},
$$

taken with the points on the boundary of $D_{z}$ which satisfy

$$
p^{2}=a^{2}, \quad a^{2}-e^{2}<q^{2} \leqq a^{2}+e^{2},
$$

form two continuous curve segments which correspond under $T$ to those two sides of the rectangle $E_{2}$ which satisfy

$$
u^{2}=a^{2}, \quad v^{2} \leqq b^{2} .
$$

The points $(p q)$ satisfying (115) are the two finite curve segments cut off from the hyperbola $p^{2}-q^{2}=e^{2}$ by the straight lines $p= \pm a$, while the points $(p q)$ satisfying (116) make up the four finite segments of the straight lines $p= \pm a$ which lie between the two conjugate hyperbolas.

(D) The points on the bonndary of $D_{2}$ which satisfy

$$
q^{2}-p^{2}=e^{2}, \quad p^{2}<a^{2},
$$

correspond under $T$ to those two sides of the rectangle $E_{\searrow}$ which satisfy

$$
u^{2}=b^{2}, \quad v^{2}<a^{2} \text {. }
$$


The points $(p q)$ satisfying (118) obviously make up those two finite segments of the hyperbola $q^{2}-p^{2}=e^{2}$ which lie between the two straight lines $p= \pm a$.

The results just obtained properly interpreted will give us the following lemma. In this lemma we will again understand by $p^{2}$ and $q^{2}$ the expressions

$$
\begin{aligned}
& p^{2}=y_{1}^{2}+y_{2}^{2}+\cdots+y_{k}^{2} \\
& q^{2}=y_{k+1}^{2}+y_{k+2}^{2}+\cdots+y_{n}^{2}
\end{aligned}
$$

and in addition, in terms of the variables $\left(z_{1}, z_{2}, \ldots, z_{n}\right)=(Z)$ we set

$$
\begin{aligned}
& u^{2}=z_{1}^{2}+z_{2}^{2}+\cdots+z_{k}^{2} \\
& v^{2}=z_{k+1}^{2}+z_{k+2}^{2}+\cdots+z_{n}^{2}
\end{aligned}
$$

Lemma 9. There exists a one-to-one continuous correspondence between $a$ set of points $\left(y_{1}, y_{2}, \cdots, y_{n}\right)=(Y)$ and a set of points $\left(z_{1}, z_{2}, \cdots, z_{n}\right)=(Z)$ (a) under which the set of points $(Y)$ satisfying

$$
-e^{2} \leqq q^{2}-p^{2} \leqq e^{2}, \quad p^{2} \leqq a^{2}
$$

corresponds to the points $(Z)$ satisfying

$$
u^{2} \leqq a^{2}, \quad v^{2} \leqq b^{2}
$$

(b) while that part of the boundary of (122). that consists of points $(Y)$ satisfying

$$
p^{2}-q^{2}=e^{2}, \quad q^{2} \leqq a^{2}-e^{2}
$$

and of points $(Y)$ satisfying

$$
p^{2}=a^{2}, \quad a^{2}-e^{2}<q^{2} \leqq a^{2}+e^{2}
$$

corresponds to those boundary points (Z) of (123) which satisfy

$$
u^{2}=a^{2}, \quad v^{2} \leqq b^{2}
$$

(c) while the boundary points $(Y)$ of (122) satisfying

$$
q^{2}-p^{2}=e^{2}, \quad p^{2}<a^{2}
$$


correspond to those boundary points $(Z)$ of (123) which satisfy

$$
v^{2}=b^{2}, \quad u^{2}<a^{2} .
$$

The correspondence whose existence is affirmed in this lemma may be given in terms of the function $r\left(p^{2} q^{2}\right)$ of (111) as follows:

$$
z_{i}=r\left(p^{2} q^{2}\right) y_{i} \quad(i=1,2, \cdots, n),
$$

where $p^{2}$ and $q^{2}$ here as in the future stand for the functions of the $y$ 's to which they are set equal in (120).

From (129) we have at once that

$$
\begin{aligned}
& {\left[z_{1}^{2}+z_{2}^{2}+\cdots+z_{l k}^{2}\right]=r^{2}\left(p^{2} q^{2}\right)\left[y_{1}^{2}+y_{2}^{2}+\cdots+y_{k}^{2}\right],} \\
& {\left[z_{k+1}^{2}+\cdots+z_{n}^{2}\right]=r^{2}\left(p^{2} q^{2}\right)\left[y_{k+1}^{2}+\cdots+y_{n}^{2}\right] .}
\end{aligned}
$$

Equations (130) show that the functions $p^{2}$ and $q^{2}$ are related under the transformation (129) to the functions $u^{2}$ and $v^{2}$ by equations obtained by squaring the different members of (111). But from equations (111) so squared follow those parts of the results of (B), (C) and (D) which describe in detail how $p^{2}$ and $q^{2}$ are transformed into $u^{2}$ and $v^{2}$, and the analytical results there contained abstracted from their geometric setting lead directly to the results (a), (b) and (c) of this lemma.

The correspondence is obviously continuous. That it is one-to-one will now be proved by giving its inverse. From (111) and (112) it follows that if neither $(p q)$ nor $(u v)=(00)$

$$
\frac{1}{r\left(p^{2} q^{2}\right)}=\sigma\left(u^{2} v^{2}\right) .
$$

Thus (129) may be written

$$
y_{i}=\sigma\left(u^{2} v^{2}\right) z_{i} \quad(i=1,2, \cdots, n),
$$

where here $u^{2}$ and $v^{2}$ stand for those functions of the $z$ 's to which they are set equal in (121). Further, (131) obviously also holds for the points just excepted, namely $(Y)=(Z)=(0,0, \ldots, 0)$. Thus the correspondence is one-to-one, and the lemma is completely proved.

The set of points $(Z)$ satisfying (123) can readily be shown to make up an $n$-cell and its boundary. Hence the corresponding set of points $(Y$, satisfying (122) make up an $n$-cell and its boundary. The set of points $(Y)$ satisfying (122) has been termed the set $D_{n} . D_{n}$ will also be used to 
designate the set of points $(X)=\left(x_{1}, x_{2}, \cdots, x_{n}\right)$ corresponding to the points $(Y)$ of $D_{n}$.

Let $c$ be the value of $f$ at the given critical point. All of the points $(X)$ of $D_{n}$ satisfy

$$
f \leqq c+e^{2}
$$

and are separated from the remaining points $(X)$ satisfying (132) by those boundary points of $D_{n}$ satisfying (124) and (125). These boundary points of $D_{n}$ satisfying (124) and (125) will be termed the old boundary points of $D_{n}$ while the remaining boundary points of $D_{n}$, namely those satisfying (127), will be termed the new boundary points of $D_{n}$. The set of all points of $D_{n}$ other than the old boundary points of $D_{n}$ will be called the new points of $D_{n}$.

The points $(Z)$ satisfying (123) may be represented by a pair of points $(P Q)$ of which $P$ is a point in a $l_{i}$-dimensional space with coordinates $\left(z_{1}, z_{z}, \cdots, z_{k}\right)$ satisfying

$$
u^{2} \leqq a^{2}
$$

and $Q$ is a point in an $(n-k)$-dimensional space with coördinates $\left(z_{k+1}, z_{k+2}, \cdots, z_{n}\right)$ satisfying

$$
v^{2} \leqq b^{2}
$$

Let the $(k-1)$-dimensional hypersphere $u^{2}=a^{2}$ in the space of the points $P$ be denoted by $S_{k-1}$ and the $(n-k-1)$-dimensional hypersphere $v^{2}=b^{2}$ in the space of the points $Q$ be denoted by $S_{n-k-1}$. With the above convention the results of Lemma 9 may be translated into the following lemma.

Lemma 10. The set $D_{n}$ of points ( $Y$ ) satisfying (122) make up an $n$-cell and its boundary. It can be put into one-to-one continuous correspondence with the set of pairs of points $(P Q)$ obtained by combining an arbitrary point $P$ interior to or on $a(k-1)$-dimensional hypersphere $S_{k-1}$ with an arbitrary point $Q$ interior to or on an $(n-k-1)$-dimensional hypersphere $S_{n-k-1}$. In this correspondence, points on the old boundary of $D_{n}$ correspond to those pairs $(P Q)$ that are obtained by combining an arbitrary point $P$ on $S_{k-1}$ with an arbitrary point $Q$ interior to or on $S_{n-k-1}$, while points on the new boundary of $D_{n}$ correspond to those pairs of $(P Q)$ that are obtained by combining an arbitrary point $P$ interior to $S_{k-1}$ with an arbitrary point $Q$ on $S_{n-k-1}$.

If we make use of the terms of the preceding lemma, Lemma 8 may be restated and completed as follows. 
Lemma 11. The complex of points satisfying $f \leqq c-e^{2}$ can be put into one-to-one continuous correspondence with the complex of points, say $C_{n}^{\prime}$, consisting of the points satisfying $f \leqq c+e^{2}$ with the new points of $D_{n}$ excluded. The remaining points of $D_{n}$, namely the points on the old boundary of $D_{n}$, are on the boundary of $C_{n}^{\prime}$.

14. A representation of $D_{n}$ in terms of the cells of $S_{k-1}$ and $S_{n-k-1}$. Suppose the hypersphere $S_{k-1}$ "covered just once" by a nonsingular complex whose matrices of incidence $\mathrm{H}_{i}$ are given by the equations

$$
\mathrm{H}_{i}=\left|\begin{array}{ll}
1 & 1 \\
1 & 1
\end{array}\right| \quad(i=1,2, \cdots, k-1) .
$$

Denote the two $i$-cells of $S_{k-1}$ by $a_{i}^{\prime}$ and $a_{i}^{\prime \prime}(i=0,1, \ldots, k-1)$. Denote the $k$-cell consisting of the points interior to $S_{k-1}$ by $a_{k}$. Similarly suppose $S_{n-k-1}$ covered just once by a non-singular complex whose matrices of incidence $\mathrm{H}_{j}(j=1,2, \ldots, n-k-1)$ are each identical with the preceding matrices $\mathrm{H}_{i}$. Denote the two $j$-cells of $S_{n-k-1}$ by $b_{j}^{\prime}$ and $b_{j}^{\prime \prime}$ $(j=0,1, \cdots, n-k-1)$. Denote the $(n-k)$-cell consisting of the points interior to $S_{n-k-1}$ by $b_{n-k}$.

Let $\alpha$ be any one of the cells $a_{i}^{\prime}, a_{i}^{\prime \prime}$ or $a_{k}$, and $\beta$ be any one of the cells $b_{j}^{\prime}, b_{j}^{\prime \prime}$, or $b_{n-k}$. If an arbitrary point $P$ of a cell $\alpha$ be combined with an arbitrary point $Q$ of a cell $\beta$ there will result a set of pairs of points $(P Q)$ which may be considered as the elements of an $(i+j)$-cell. Denote such an $(i+j)$-cell by $(\alpha \beta)$. According to Lemma 10 the complex consisting of all such $(i+j)$-cells will be a non-singular complex covering $D_{n}$ just once. This complex of $(i+j)$-cells will be adopted as the future representation of $D_{n}$.

It follows from Lemma 10 that in the representation of $D_{n}$ just adopted the interior points of $D_{n}$ will be represented by the $n$-cell $\left(a_{k} b_{n-k}\right)$ while the new boundary of $D_{n}$ will be represented by the $(k+j)$-cells $\left(a_{k} b_{j}^{\prime}\right)$ and $\left(a_{k} b_{j}^{\prime \prime}\right)$. Here $k$ is the number specifying the type of critical point being considered, and $j$ takes on the values $0,1, \ldots, n-k-1$. It should be noted that for each value of $j$ there are just two of these $(k+j)$-cells. The cells $\left(a_{k} b_{j}^{\prime}\right),\left(a_{k} b_{j}^{\prime \prime}\right)$, and $\left(a_{k} b_{n-k}\right)$ will be termed the new cells of $D_{n}$ and the remaining cells of $D_{n}$ the old cells of $D_{n}$.

The above representation of $D_{n}$ shows that there are no new cells of dimensionality less than $k$. The points on the boundary of a cell $(\boldsymbol{\alpha} \beta)$ will be represented by the pairs $(P Q)$ obtained by combining a point $P$ on the boundary of $\alpha$ with a point $Q$ on $\beta$ or its boundary, or by combining a point $Q$ on the boundary of $\beta$ with a point $P$ on $\alpha$ or its boundary. For the work to follow it is important to determine the $(m-1)$-cells on 
the boundary of each new $m$-cell. We begin with the new cells of lowest dimensionality.

The only $(k-1)$-cells on the boundary of the new $k$-cell $\left(a_{k} b_{0}^{\prime}\right)$ will be obtained by combining an arbitrary point of a boundary $(k-1)$-cell of $a_{k}$, namely $a_{k-1}^{\prime}$ or $a_{k-1}^{\prime}$, with $b_{0}^{\prime}$ giving thus the two $(k-1)$-cells

$$
\left(a_{k-1}^{\prime} b_{0}^{\prime}\right),\left(a_{k-1}^{\prime \prime} b_{0}^{\prime}\right) \text { on }\left(a_{k} b_{0}^{\prime}\right),
$$

both of which are old cells of $D_{n}$. Similarly the only $(k-1)$-cells on the boundary of $\left(a_{k} b_{0}^{\prime \prime}\right)$ are the old cells

$$
\left(a_{k-1}^{\prime} b_{0}^{\prime \prime}\right),\left(a_{k-1}^{\prime \prime} b_{0}^{\prime \prime}\right) \text { on }\left(a_{k} b_{0}^{\prime \prime}\right) .
$$

For $j=1,2, \cdots, n-k-1$ the $(k+j-1)$-cells on the boundary of the new $(k+j)$-cell $\left(a_{k} b_{j}^{\prime}\right)$ are obtained by combining a boundary $(k-1)$-cell of $a_{k}$, namely $a_{k-1}^{\prime}$ or $a_{k-1}^{\prime \prime}$; with $b_{j}^{\prime}$, or by combining a boundary $(j-1)$-cell of $b_{j}^{\prime}$, namely $b_{j-1}^{\prime}$ or $b_{j-1}^{\prime \prime}$, with $a_{k}$, giving thus the $(k+j-1)$-cells

$\left(a_{k-1}^{\prime} b_{j}^{\prime}\right),\left(a_{k-1}^{\prime \prime} b_{j}^{\prime}\right),\left(a_{k} b_{j-1}^{\prime}\right),\left(a_{k} b_{j-1}^{\prime \prime}\right)$ on $\left(a_{k} b_{j}^{\prime}\right) \quad(j=1,2, \ldots, n-k-1)$,

of which the first two cells are old cells and the latter two cells are new cells. Similarly the $(k+j-1)$-cells on the boundary of the new $(k+j)$-cell $\left(a_{k} b_{j}^{\prime \prime}\right)$ are the cells

$$
\left(a_{k-1}^{\prime} b_{j}^{\prime \prime}\right),\left(a_{k-1}^{\prime \prime} b_{j}^{\prime \prime}\right),\left(a_{k} b_{j-1}^{\prime}\right),\left(a_{k} b_{j-1}^{\prime \prime}\right) \text { on }\left(a_{k} \bar{b}_{j}^{\prime \prime}\right) \quad(j=1,2, \cdots, n-k-1),
$$

of which the first two are old cells and the latter two are new cells. Finally the $(n-1)$-cells on the boundary of the new $n$-cell $\left(a_{k} b_{n-k}\right)$ are

$$
\left(a_{k-1}^{\prime} b_{n-k}\right), \quad\left(a_{k-1}^{\prime \prime} b_{n-k}\right), \quad\left(a_{k} b_{n-k-1}^{\prime}\right), \quad\left(a_{k} b_{n-k-1}^{\prime \prime}\right) \quad \text { on } \quad\left(a_{k} b_{n-k}\right)
$$

of which the first two are old cells and the latter two are new cells.

15. Differences between the connectivity numbers of the complex $f \leqq c-e^{2}$ and the complex $f \leqq c+e^{2}$. It follows from Lemma 11 that the complex $f \leqq c-e^{2}$ has the same connectivity numbers as the complex $C_{n}^{\prime}$ consisting of the points satisfying $f \leqq c+e^{2}$ with the new points of $D_{n}$ excluded. The problem of this section then resolves itself into one of determining the relations between the connectivity numbers of the complex $C_{n}^{\prime}$ and those of the complex $f \leqq c+e^{2}$. Denote the latter complex by $C_{n}$.

If the new points of $D_{n}$ be added to $C_{n}^{\prime}$ there results $C_{n}$. The question then is what is the effect upon the connectivity numbers of adding the 
new points of $D_{n}$ to $C_{n}^{\prime}$ ? According to Lemma 11 the only points of $D_{n}$ coinciding with points of $C_{n}^{\prime}$ are the old boundary points of $D_{n}$. Because of the simple nature of the functions defining the boundaries of these complexes we can and will suppose $C_{n}^{\prime}$ broken up into its component cells in such a manner that points of $C_{n}^{\prime}$ that coincide with points of $D_{n}$ all lie on cells of $C_{n}^{\prime}$ that coincide with cells of $D_{n}$.

Let $\mathrm{H}_{i}(i=1,2, \ldots, n)$ be the $i$ th matrix of incidence of $C_{n}$. Let $\rho_{i}$ be the rank $(\bmod 2)$ of $\mathrm{H}_{i}$. For the same values of $i$ let $\mathrm{H}_{i}^{\prime}$ be the $i$ th matrix of incidence of $C_{n}^{\prime}$. Let $\varrho_{i}^{\prime}$ be the rank $(\bmod 2)$ of $\mathrm{H}_{i}^{\prime}$. Let $R_{j}(j=0,1, \ldots, n)$ be the $j$ th connectivity number of $C_{n}$ and $R_{j}^{\prime}$ that of $C_{n}^{\prime}$. For the same values of $j$ let $\alpha_{j}$ be the number of $j$-cells on $C_{n}$ and $\alpha_{j}^{\prime}$ the corresponding number for $C_{n}^{\prime}$. The following well known equations will be used:

$$
\begin{aligned}
& R_{0}-1=\alpha_{0}-\varrho_{1}-1, \\
& R_{1}-1=\alpha_{1}-\varrho_{1}-\varrho_{2}, \\
& R_{2}-1=\alpha_{2}-\varrho_{2}-\varrho_{3}, \\
& \cdot \cdot \cdot \cdot \cdot \cdot \cdot \cdot \cdot \cdot \cdot, \\
& R_{n-1}-1=\alpha_{n-1}-\varrho_{n-1}-\varrho_{n}, \\
& R_{n}-1=\alpha_{n}-\varrho_{n} .
\end{aligned}
$$

Equations (133) refer to $C_{n}$. Similar equations hold for $C_{n}^{\prime}$ and are obtained by priming each letter in (133). From equations (133) and equations (133) with the primes added there can be obtained by a subtraction of the corresponding members of the respective equalities the equations

$$
\begin{aligned}
& R_{0}-R_{0}^{\prime}=\alpha_{0}-\alpha_{0}^{\prime}-\left(\varrho_{1}-\varrho_{1}^{\prime}\right), \\
& R_{1}-R_{1}^{\prime}=\alpha_{1}-\alpha_{1}^{\prime}-\left(\varrho_{1}-\varrho_{1}^{\prime}\right)-\left(\varrho_{2}-\varrho_{2}^{\prime}\right), \\
& R_{2}-R_{2}^{\prime}=\alpha_{2}-\alpha_{2}^{\prime}-\left(\varrho_{2}-\varrho_{2}^{\prime}\right)-\left(\varrho_{3}-\varrho_{3}^{\prime}\right), \\
& \cdot \cdot \cdot \cdot \cdot \cdot \cdot \cdot \cdot \cdot \cdot \cdot \cdot \cdot \cdot \cdot \cdot \cdot \cdot \cdot \cdot \cdot \cdot \cdot \cdot \cdot \cdot \cdot{ }^{\prime} \cdot \rho_{n-1}^{\prime}-\left(\varrho_{n-1}-\varrho_{n-1}^{\prime}\right)-\left(\varrho_{n}-\varrho_{n}^{\prime}\right), \\
& \left.R_{n-1}-R_{n-1}^{\prime}=\alpha_{n-1}-\alpha_{n-1}^{\prime}\right) \\
& R_{n}-R_{n}^{\prime}=\alpha_{n}-\alpha_{n}^{\prime}-\left(\varrho_{n}-\varrho_{n}^{\prime}\right) .
\end{aligned}
$$

To apply these equations the relations between the matrices $\mathbf{H}_{i}$ and $\mathbf{H}_{\boldsymbol{i}}^{\prime}$ will now be determined. In the first place we have

$$
\mathrm{H}_{i}=\mathrm{H}_{i}^{\prime} \quad(i=1,2, \cdots, k-1),
$$

since for these values of $i$ there are no new $i$-cells $(\S 14)$ so that for these values of $i, C_{n}$ and $C_{n}^{\prime}$ contain the same $i$-cells. According to the results of $\S 14$ the table of incidence of the $(k-1)$-cells with the $k$-cells of $C_{n}$ will take the following form: 


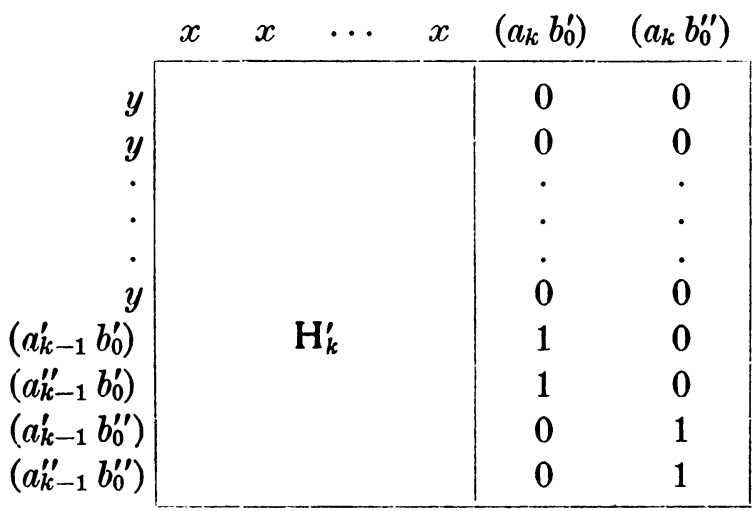

where the $x$ 's represent $k$-cells which it is not desired to give more explicitly and the $y$ 's represent $(k-1)$-cells. The rectangle containing $\mathrm{H}_{k}^{\prime}$ is supposed filled out with the elements of the matrix $\mathrm{H}_{k}^{\prime}$. The matrix $\mathrm{H}_{k}$ of course consists of the elements of the above two rectangles combined. It can be simplified as follows. The two new $k$-cells written above the last two columns are on at least one new $(k+1)$-cell $(\S 14)$. The boundary of this $(k+1)$-cell is a $k$-circuit. Let the columns, other than the last column, which in the table are written under $k$-cells that are on this k-circuit, be added to the last column. The last column will thereby be reduced to zero $(\bmod 2)$ without altering the rank of $\mathrm{H}_{k}$. Let the third from the last row be added to fourth from the last row. As a result there will be but one element not zero in the last two columns. The rank $\varrho_{k}^{\prime}$ of the matrix of elements of $\mathrm{H}_{k}^{\prime}$ has by these processes not been altered $(\bmod 2)$. It is seen that for $\varrho_{k}$ there are two possibilities depending upon the nature of the resulting matrix in the left hand rectangle, namely either

Case 1, or

Case 2,

$$
\varrho_{k}=\varrho_{k}^{\prime}
$$$$
\varrho_{k}=\varrho_{k}^{\prime}+1
$$

It may be shown by examples that both of these cases actually occur.

The tables of incidence corresponding to the matrices $\mathrm{H}_{k+j}$ for $j=1,2, \ldots, n-k-1(\S 14)$ are given by the first table on page 384 . In the special case where $k=n-1$ there is no table of this sort.

In this table conventions similar to those of the previous table are made. The matrix $\mathrm{H}_{k+j}$ is of course the matrix of all the elements included in the four rectangles. It can be simplified as follows. First, exactly as in the case of the preceding table there is a set of columns which added to the last column $(\bmod 2)$ reduce that column to zeros. If now the next to the last row be added to the three other rows that have 


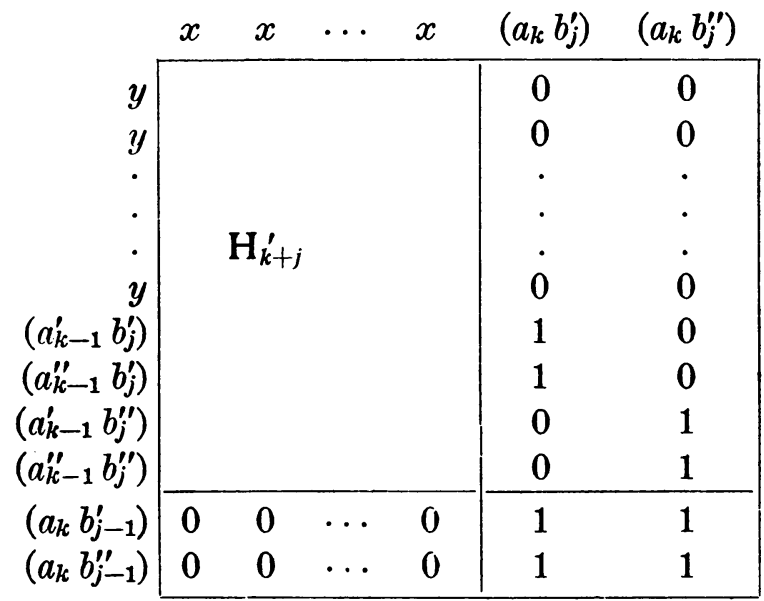

unity in the next to the last column, there will be left $(\bmod 2)$ in the last two rows and columns only one element not zero and that a 1 in the next to the last row and column. It follows that

$$
\varrho_{k+j}=\varrho_{k+j}^{\prime}+1 \quad(j=1,2, \cdots, n-k-1) .
$$

Finally, corresponding to the matrix $\mathrm{H}_{n}$ there is the table

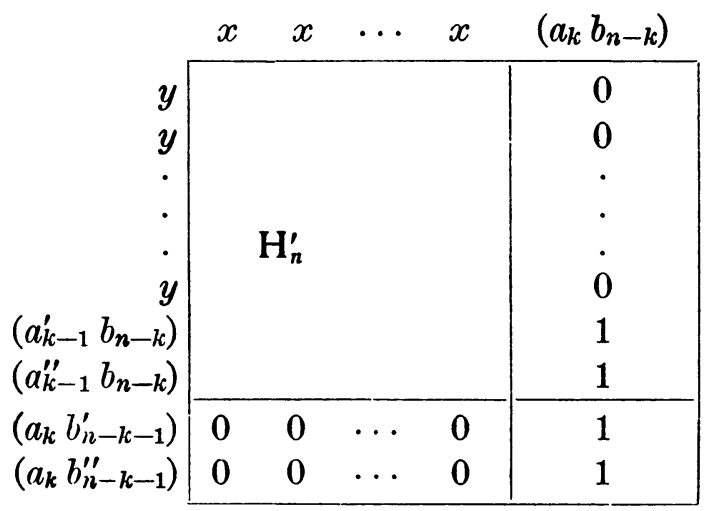

from which it follows that

$$
\varrho_{n}=\varrho_{n}^{\prime}+1
$$

The number of cells of any particular rank which are contained in $C_{n}$ but not in $C_{n}^{\prime}$ can be read off from the preceding tables. We tabulate the following results:

$$
\begin{aligned}
& \alpha_{i}-\alpha_{i}^{\prime}=0, \quad \varrho_{i}-\varrho_{i}^{\prime}=0 \\
& \alpha_{i}-\alpha_{i}^{\prime}=2, \quad \varrho_{i}-\varrho_{i}^{\prime}=0 \\
& (i=1,2, \ldots, k-1) \text {; } \\
& \alpha_{i}-\alpha_{i}^{\prime}=2, \quad \varrho_{i}-\varrho_{i}^{\prime}=1 \quad(i=k \quad \text { Case 2); } \\
& \alpha_{i}-\alpha_{i}^{\prime}=2, \quad \varrho_{i}-\varrho_{i}^{\prime}=1 \quad(i=k+1, k+2, \cdots, n-1) \text {; } \\
& \alpha_{i}-\alpha_{i}^{\prime}=1, \quad \varrho_{i}-\varrho_{i}^{\prime}=1 \quad(i=n) \text {. }
\end{aligned}
$$


From these equations and equations (134) it follows for a critical point of the $k$ th type $(0<k<n)$ that

while either

$$
R_{i}=R_{i}^{\prime} \quad(i=0,1, \cdots, k-2, k+1, k+2, \cdots, n)
$$

or

$$
R_{k-1}=R_{k-1}^{\prime} \quad \text { and } \quad h_{k}=R_{k}^{\prime}+1 \quad \text { (Case 1) }
$$

$$
R_{k-1}=R_{k-1}^{\prime}-1 \quad \text { and } \quad R_{k}=R_{k}^{\prime}
$$

(Case 2).

That both Case 1 and Case 2 are possible may be shown by simple examples. If we recall that the complex $C_{n}^{\prime}$ has the same connectivity numbers as the complex $f \leqq c-e^{2}$ (Lemma 11, §13) we have the following fundamental theorem.

THEOREm 3. Let $c$ be a critical value assumed by $f$ at just one critical point and that a critical point of a type for which $0<k<n$; then for a sufficiently small positive constant e the connectivity numbers $R_{i}$ of the complex of points satisfying $f \leqq c+e^{2}$ differ from the connectivity numbers $R_{i}^{\prime}$ of the complex of points satisfying $f \leqq c-e^{2}$ only in that either

or else

$$
R_{k}=R_{k}^{\prime}+1
$$

$$
R_{k-1}=R_{k-1}^{\prime}-1
$$

\section{CRITICAL POINTS OF THE $n$ TH TYPE}

16. Let there be given a critical value of the given function, say $c$, taken on by $f$ at a critical point of the $n$th type, and at no other critical point. Let $e$ be a positive constant so small that there is no critical value, other than $c$, between $c-e^{2}$ and $c+e^{2}$, or equal to $c-e^{2}$ or $c+e^{2}$. This section is devoted to proving the following theorem.

THEOREm 4. If c be a critical value of $f$ taken on at just one critical point, and that of the nth type, then for a sufficiently small positive constant e, the connectivity numbers $R_{i}$ of the complex of points in $S$ satisfying

$$
f \leqq c+e^{2}
$$

differ from the connectivity numbers $R_{i}^{\prime}$ of the complex of points in $S$ satisfying

only in that

$$
f \leqq c-e^{2}
$$

$$
R_{n-1}=R_{n-1}^{\prime}-1 \text {. }
$$

Before proving this theorem the following lemma will be established.

Lemma. For any complex $C_{n}$ lying in a finite part of euclidean $n$-space, and bounded by a finite number of distinct closed manifolds on each of 
which $f$ takes on a non-critical constant value the connectivity number $R_{n}$ equals unity.

While a theorem similar to this could be proved under much more general hypotheses this particular lemma is all that we need here. To prove the lemma observe that in the given euclidean $n$-space we can choose an $(n-1)$-dimensional hypersphere $S_{n-1}$ so large that it contains $C_{n}$ entirely in its interior. Let $A_{n}$ be the complex consisting of the points interior to and on $S_{n-1} . A_{n}$ can be broken up into component cells in such a manner as to contain $C_{n}$ as a sub-complex of its cells. Now as is well known, for $A_{n}, R_{n}=1$, or in equivalent terms $A_{n}$ contains no $n$-circuits. Hence $C_{n}$ contains no $n$-circuits, and its connectivity number $R_{n}$ must also be unity. Thus the lemma is proved.

In terms of the $y$ 's of Lemma $4, \S 3, f$ can be represented in the neighborhood of the given critical point in the form

$$
f-c=-y_{1}^{2}-y_{2}^{2}-\cdots-y_{n}^{2} .
$$

If the points $(Y)$ satisfy

$$
0 \leqq y_{1}^{2}+y_{2}^{2}+\cdots+y_{n}^{2}<e^{2},
$$

the corresponding points $(X)$ satisfy the relations

$$
c-e^{2}<f \leqq c
$$

and thus satisfy (135) but not (136).

Denote the complex (135) by $C_{n}$ and the complex (136) by $C_{n}^{\prime}$. The points $(X)$ corresponding to the points $(Y)$ satisfying (137) obviously make up an $n$-cell, say $a_{n}$, which belongs to $C_{n}$. The boundary of $a_{n}$ consists of points at which the sum of the squares of the $y$ 's equals $e^{2}$ and so consists of points at which $f=c-e^{2}$. The boundary of $a_{n}$ thus lies on the boundary of $C_{n}^{\prime}$. Let $a_{n}$ be added to $C_{n}^{\prime}$, thereby forming a complex denoted by $C_{n}^{\prime \prime}$. If use be made of the orthogonal trajectories employed for a similar purpose in $\S 7$, it is easy to prove that $C_{n}$ and $C_{n}^{\prime \prime}$ can be put into one-to-one continuous correspondence and hence have the same connectivity numbers $\mathrm{R}^{i}$.

The question remains as to what is the relation between the connectivity numbers $R_{i}$ of $C_{n}^{\prime \prime}$ and those $R_{i}^{\prime}$ of $C_{n}^{\prime}$. $C_{n}^{\prime}$ and $C_{n}^{\prime \prime}$ differ only in that $a_{n}$ belongs to $C_{n}^{\prime \prime}$ and not to $C_{n}^{\prime}$. If then $C_{n}^{\prime \prime}$ be supposed represented by the cells of $C_{n}^{\prime}$ together with the $n$-cell $a_{n}$, it appears that all the matrices of incidence of $C_{n}^{\prime}$ and of $C_{n}^{\prime \prime}$ will be identical except the $n$th ones. From formulas (134) it follows that the connectivity numbers of $C_{n}^{\prime}$ and $C_{n}^{\prime \prime}$ are 
the same with the possible exception of the $n$th and $(n-1)$ th connectivity numbers.

Now it follows from the preceding lemma that both $R_{n}$ and $h_{n}^{\prime}$ equal unity so that

$$
R_{n}-R_{n}^{\prime}=0
$$

Let $\alpha_{i}^{\prime}$ and $\alpha_{i}^{\prime \prime}$ be respectively the numbers of $i$-cells in $C_{n}^{\prime}$ and $C_{n}^{\prime \prime}$, and $\varrho_{j}^{\prime}$ and $\varrho_{j}^{\prime \prime}$ be respectively the ranks of the $j$ th matrix of incidence of $C_{n}^{\prime}$ and $C_{n}^{\prime \prime}$. Since $a_{n}$ is the only $n$-cell in $C_{n}^{\prime \prime}$ that is not in $C_{n}^{\prime}$ we have

$$
\alpha_{n}^{\prime \prime}-\alpha_{n}^{\prime}=1 \text {. }
$$

The last two equations of (134) become under the present notation

$$
\begin{aligned}
& R_{n-1}-R_{n-1}^{\prime}=\alpha_{n-1}^{\prime \prime}-\alpha_{n-1}^{\prime}-\left(\varrho_{n-1}^{\prime \prime}-\varrho_{n-1}^{\prime}\right)-\left(\varrho_{n}^{\prime \prime}-\varrho_{n}^{\prime}\right), \\
& R_{n}-R_{n}^{\prime}=\alpha_{n}^{\prime \prime}-\alpha_{n}^{\prime}-\left(\varrho_{n}^{\prime \prime}-\varrho_{n}^{\prime}\right) .
\end{aligned}
$$

From (138), (139) and (141) it follows that

$$
\varrho_{n}^{\prime \prime}-\varrho_{n}^{\prime}=1 \text {. }
$$

Since $\alpha_{n-1}^{\prime \prime}=\alpha_{n-1}^{\prime}$ and $\varrho_{n-1}^{\prime \prime}=\varrho_{n-1}^{\prime}$ it follows from (140) that

$$
R_{n-1}=R_{n-1}^{\prime}-1
$$

Thus the theorem is completely proved.

\section{General theorems Concerning the CRITICAL POINTS}

17. The case of a critical value of $f$ taken on at several different critical points. Up to this point only those critical values of $f$ have been considered which are taken on at just one critical point. A first theorem in the general case is the following:

Theorem 5. Let $c$ be any critical value of $f$ that is not the absolute minimum of $f$ in $S$, and $m_{i}$ be the number of critical points of the ith type at which $f=c$. Let $e$ be a small positive constant. Let $R_{i}$ and $R_{i}^{\prime}$ be respectively the ith connectivity numbers of the complex of points in $S$ satisfying

$$
f \leqq c+e^{2}
$$

and

$$
f \leqq c-e^{2}
$$

Then for $e$ sufficiently small there exist integers $p_{0}, p_{1}, \cdots, p_{n-1}$ and $q_{1}, q_{2}, \cdots, q_{n}$, all positive or zero, such that 
and such that

$$
\begin{aligned}
& m_{0}=p_{0}, \\
& m_{1}=p_{1}+q_{1}, \\
& m_{2}=p_{2}+q_{2}, \\
& \cdot \cdot \cdot \cdot \cdot \cdot \cdot \\
& m_{n-1}=p_{n-1}+q_{n-1}, \\
& m_{n}=q_{n},
\end{aligned}
$$

while, as ever,

$$
\begin{aligned}
& R_{0}-R_{0}^{\prime}=p_{0}-q_{1}, \\
& R_{1}-R_{1}^{\prime}=p_{1}-q_{2}, \\
& \cdot \cdot \cdot \cdot \cdot \cdot \cdot \cdot \cdot \cdot \\
& R_{n-1}-R_{n-1}^{\prime}=p_{n-1}-q_{n},
\end{aligned}
$$

$$
R_{n}=R_{n}^{\prime}=1
$$

Denote the complex defined by (143) by $C_{n}$, and that defined by (144) by $C_{n}^{\prime}$. The critical points at which $f=c$ are divided for convenience into those of type 0 , those of type $n$, and those of the remaining types. Corresponding to this division of the critical points into classes the proof of the above theorem is divided into parts $\mathrm{A}, \mathrm{B}$, and $\mathrm{C}$.

A. Critical points of type 0 at which $f=c$. For $e$ sufficiently small it follows exactly as in the proof of Theorem 2 of $\S 9$ that the points in $C_{n}$ neighboring the $m_{0}$ critical points of type 0 make up $m_{0} n$-cells and their boundaries, distinct from each other and from any other points of $C_{n}$, and not included at all among the points of $C_{n}^{\prime}$. If these $m_{0} n$-cells and their boundaries be added to $C_{n}^{\prime}$ there will result a complex, say $C_{n}^{\prime \prime}$, whose connectir ' $\mathrm{y}$ numbers $R_{n}^{\prime \prime}$ will differ from those of $C_{n}^{\prime}$ only in that

$$
R_{0}^{\prime \prime}-R_{0}^{\prime}=m_{0} \text {. }
$$

B. Critical points of type $n$ at which $f=c$. For $e$ sufficiently small it follows exactly as in the proof of Theorem $4, \S 16$, that the points in the neighborhood of the $m_{n}$ critical points of type $n$ make up $m_{n}$ distinct $n$-cells whose boundaries belong to $C_{n}^{\prime}$, but which themselves belong only to $C_{n}$. If these $m_{n} n$-cells be added to $C_{n}^{\prime \prime}$ there will result a complex $C_{n}^{\prime \prime \prime}$ whose connectivity numbers $R_{i}^{\prime \prime \prime}$ will differ from those of $C_{n}^{\prime \prime}$ only in that

$$
R_{n-1}^{\prime \prime \prime}-R_{n-1}^{\prime \prime}=-m_{n} \text {. }
$$

C. Critical points not of type 0 or $n$ at which $f==$ a. With each critical point of this class there can be associated a complex of points $D_{n}$, exactly as is done in Lemmas 10 and 11 for the case where the given 
critical point is the only critical point at which $f=c$. For $e$ and $a$ sufficiently small the different complexes $D_{n}$ will all be distinct from each other. The so called "new points" of each complex $D_{n}$ will belong to $C_{n}$. Suppose that there are just $r$ critical points, at which $f=c$ and which are of a type neither 0 or $n$. Let $A_{j}$ be the set of new points in the complex $D_{n}$ associated with the $j$ th one of these critical points $(j=1,2, \ldots, r)$. Let all of the points of $C_{n}$ not already in $C_{n}^{\prime \prime \prime}$ and not among the points of any set $A_{j}$ be added to $C_{n}^{\prime \prime \prime}$, thereby forming a complex $C_{n}^{\prime \prime \prime \prime}$. $C_{n}^{\prime \prime \prime \prime}$ and $C_{n}^{\prime \prime \prime}$ can be put into one-to-one continuous correspondence by the methods of Lemma $11, \S 13$, so that the complexes $C_{n}^{\prime \prime \prime \prime}$ and $C_{n}^{\prime \prime \prime}$ will have the same connectivity numbers.

Finally to obtain $C_{n}$ we have only to add to $C_{n}^{\prime \prime \prime \prime}$ the different sets $A_{j}$. We will suppose that these sets $A_{j}$ are added in the order of their subscripts. Suppose that $A_{j}$ was associated with a critical point of type $k_{j}$. The addition of $A_{j}$ to what we shall call the old complex, consisting of $C_{n}^{\prime \prime \prime \prime}$ and the points of $A_{1}, A_{2}, \cdots, A_{j-1}$, will give a new complex whose connectivity numbers will differ from the connectivity numbers of the old complex, either (Case 1) in that the $k_{j}$ th connectivity number of the new complex will be one greater than that of the old complex, or else (Case 2) the $\left(k_{j}-1\right)$ th connectivity number of the new complex will be one less than that of the old complex. We now define $p_{i}$, for $i=1,2, \cdots, n-1$, as the number of critical points of type $i$, at which $f=z$, which come under Case 1, and define $q_{i}$ for the same values of $i$ as the number of critical points of the $i$ th type at which $f=c$ which come under Case 2. The connectivity numbers $R_{i}^{\prime \prime \prime}$ of $C_{n}^{\prime \prime \prime \prime}$ are obviously then related to the connectivity numbers $R_{i}$ of $C_{n}$ as follows:

$$
\begin{aligned}
& R_{0}-R_{0}^{\prime \prime \prime}=-q_{1}, \\
& R_{1}-R_{1}^{\prime \prime \prime}=p_{1}-q_{2}, \\
& \cdot \cdot \cdot \cdot \cdot \cdot \cdot \cdot \cdot \\
& R_{n-2}-R_{n-2}^{\prime \prime \prime}=p_{n-2}-q_{n-1}, \\
& R_{n-1}-R_{n-1}^{\prime \prime \prime}=p_{n-1} .
\end{aligned}
$$

We now define $p_{0}$ as equal to $m_{0}$ and define $q_{n}$ as equal to $m_{n}$. If use be made of the fact that the connectivity numbers of $C_{n}^{\prime}$ and $C_{n}^{\prime \prime}$ have no differences other than that given by (148), and that the connectivity numbers of $C_{n}^{\prime \prime}$ and $C_{n}^{\prime \prime \prime}$ have no differences other than that given by (149), while $R_{i}^{\prime \prime \prime}=R_{i}^{\prime \prime \prime}$ without exception, then from equations (150) and the definitions of the $p$ 's and the $q$ 's the theorem follows.

18. Relations between the connectivity numbers of the complex $f \leqq c_{1}$ and the complex $f \leqq c_{2}$. 
THEOREM 6. Let $c_{1}$ and $c_{2}\left(c_{1}<c_{2}\right)$ be two non-critical values taken on by $f$ in $S$. Let $m_{i}$ be the number of critical points of the ith type at which $f$ equals some constant between $c_{1}$ and $c_{2}$. Let $R_{i}$ and $R_{i}^{\prime}$ be the ith connectivity numbers of the complexes of points in $S$ respectively satisfying $f \leqq c_{2}$ and $f \leqq c_{1}$; then there exist integers, $p_{0}, p_{1}, \cdots, p_{n-1}$, and $q_{1}, q_{2}, \cdots, q_{n}$, all positive or zero, such that

and such that

$$
\begin{aligned}
& m_{0}=p_{0}, \\
& m_{i}=p_{i}+q_{i} \quad(i=1,2, \cdots, n-1), \\
& m_{n}=q_{n},
\end{aligned}
$$

$$
R_{i}-R_{i}^{\prime}=p_{i}-q_{i+1} \quad(i=0,1, \ldots, n-1)
$$

while as ever

$$
R_{n}=R_{n}^{\prime}=1 \text {. }
$$

Let $a_{1}, a_{2}, \ldots, a_{r}$ be the critical values of $f$ between $c_{1}$ and $c_{2}$, taken in the order of increasing magnitude. With each of these critical values of $f$, say $a_{j}$, let there be associated a positive constant $e_{j}$ taken so small that for this choice of $e_{j}$ Theorem 5 holds if $c$ and $e$ in Theorem 5 are here taken to be $a_{j}$ and $e_{j}$ respectively. Let the constants $e_{j}$ be also taken so small that the constants

$$
c_{1}, \quad a_{1}-e_{1}, a_{1}+e_{1}, a_{2}-e_{2}, a_{2}+e_{2}, \cdots, a_{r}-e_{r}, a_{r}+e_{r}, c_{2}
$$

are none of them equal and appear in (151) in the order of increasing magnitude. Now there are no critical values of $f$ between $c_{1}$ and $a_{1}-e_{1}$. It follows from Lemma $6, \S 7$, that the complex $f \leqq c_{1}$ has the same counectivity numbers as the complex $f \leqq a_{1} \dot{-} e_{1}$. For the same reason the complexes $f \leqq a_{i}+e_{i}$ and $f \leqq a_{i+1}-e_{i+1}$, for $i=1,2, \ldots, r-1$, have the same connectivity numbers, as well as the complexes $f \leqq a_{r}+e_{r}$ and $f \leqq c_{2}$. The relations between the comnectivity numbers of the complex $f \leqq a_{j}+e_{j}$ and the complex $f \leqq a_{j}-c_{j}$ are of the nature of those given by the preceding Theorem 5 .

If then there be considered in succession the complexes of points in $S$ satisfying $f \leqq c$, where $c$ takes on, in succession, the constants in (151) in their order from left to right, the relations between these successive complexes will combine into the relations of which this theorem affirms the existence.

19. Relations between all of the critical points in $S$ and the connectivities of $S$. The following theorem is proved under the boundary conditions $\alpha$ of $\S 4$.

THEOREM 7. Let $M_{i}$ be the total number of critical points of $f$ of the ith type $(i=0,1, \ldots, n)$. Let $R_{i}$ be the $j$ th connectivity number of $S$. 
Under the boundary conditions a there exist integers $P_{0}, P_{1}, \ldots, P_{n-1}$ and $Q_{1}, Q_{2}, \cdots, Q_{n}$, all positive or zero, such that

$$
\begin{aligned}
& M_{0}=P_{0}+1, \\
& M_{i}=P_{i}+Q_{i}
\end{aligned} \quad(i=1,2, \cdots, n-1),
$$

and such that

while as ever

$$
R_{i}-1=P_{i}-Q_{i+1} \quad(i=0,1, \ldots, n-1)
$$

Let $s$ be the number of critical points at which $f$ takes on its absolute minimum $m$. According to Theorem $2, \S 9$, for a sufficiently small positive constant $e$, the connectivity numbers $R_{i}^{\prime}$ of the complex of points satisfying $f \leqq m+e^{2}$ are given by the relations

$$
R_{0}^{\prime}=s, \quad R_{1}^{\prime}=R_{2}^{\prime}=\ldots=R_{n}^{\prime}=1 .
$$

The proof of these results in Theorem 2 presupposed that $e$ was so small that there were no other critical points than the $s$ absolute minimum points of $f$ among the points in $S$ satisfying $f \leqq m+e^{2}$. This supposition is again made here. For this choice of $e$, an application can now be made of Theorem 6 . In this application the notation of Theorem 6 will be taken over. The constants $c_{1}$ and $c_{2}$ of Theorem 6 will here be taken respectively as $m+e^{2}$ and the value $M$ which $f$ takes on upon the boundary of $S$. Observe now that $M_{0}$, the total number of critical points of type 0 in $S$, equals $s+m_{0}$. This we write

Otherwise

$$
m_{0}=M_{0}-s .
$$

$$
m_{i}=M_{i} \quad(i=1,2, \ldots, n) .
$$

We now give the following definitions of $P_{0}, P_{1}, \ldots, P_{n-1}$ and of $Q_{1}, Q_{2}, \cdots, Q_{n}$ :

$$
\begin{aligned}
P_{0} & =p_{0}+s-1, & & \\
P_{i} & =p_{i} & & (i=1,2, \cdots, n-1), \\
Q_{j} & =q_{j} & & (j=1,2, \cdots, n),
\end{aligned}
$$

and note that $P_{0}$, in particular, as defined, is either positive or zero, since $p_{0}$ is at least zero, and $s$ at least one.

If in the relations of Theorem 6 , for the constants $R_{i}^{\prime}$ there be substituted their values as given by (155), and for $m_{0}$ and $m_{i}$ their values from (156) 
and (157) and for $p_{0}$ and the $p_{i}$ and $q_{j}$ their values as given by (158), there will result the equations which are to be proved.

The following theorem gives the answer to one of the fundamental questions with which this paper is concerned.

THEOREM 8. Under the boundary conditions a the following relations exist between the set of numbers $M_{i}$, where $M_{i}$ is the number of critical points of the ith type of $f$ in $S$, and the set of connectivity numbers $R_{j}$ of $S$ $(i, j=0,1, \cdots, n)$ :

$$
\begin{aligned}
1 & \leqq\left(M_{0}-R_{0}+1\right), \\
1 & \geqq\left(M_{0}-R_{0}+1\right)-\left(M_{1}-R_{1}+1\right), \\
1 & \leqq\left(M_{0}-R_{0}+1\right)-\left(M_{1}-R_{1}+1\right)+\left(M_{2}-R_{2}+1\right), \\
1 & \geqq\left(M_{0}-R_{0}+1\right)-\left(M_{1}-R_{1}+1\right)+\left(M_{2}-R_{2}+1\right)-\left(M_{3}-R_{3}+1\right), \\
& \cdot \cdot \cdot \cdot \cdot \cdot \cdot \cdot \cdot \cdot \cdot \cdot \cdot \cdot \cdot \cdot \cdot \cdot \cdot \cdot \cdot \cdot \cdot \cdot \cdot \cdot \cdot \cdot \cdot \\
1 & \leqq\left[\left(M_{0}-R_{0}+1\right)-+\cdot \cdot+(-1)^{n-1}\left(M_{n-1}-R_{n-1}+1\right)\right](-1)^{n-1}, \\
1 & =\left(M_{0}-R_{0}+1\right)-+\cdots+(-1)^{n}\left(M_{n}-R_{n}+1\right) \cdot
\end{aligned}
$$

To prove the theorem consider equations (152) together with equations (153), without the last equation of (152). These equations together form the system

$$
\begin{array}{rlrl}
M_{0} & =P_{0}+1, \\
M_{i} & =P_{i}+Q_{i} & & (i=1,2, \ldots, n-1), \\
R_{j}-1 & =P_{j}-Q_{j+1} & (j=0,1, \ldots, n-1) .
\end{array}
$$

Suppose the left hand members of (159) known constants, and the $P$ 's and $Q$ 's in the right hand members unknown constants or variables. The determinant of the coefficients of these unknown variables is readily seen to be different from zero, so that if the $M$ 's and the connectivity numbers are known, the equations (159) uniquely determine the $P$ 's and $Q$ 's as constants satisfying (159). In particular the equations (159) can be solved successively for the $Q$ 's as follows:

$$
\begin{aligned}
& 1+Q_{1}=\left(M_{0}-R_{0}+1\right), \\
& 1-Q_{2}=\left(M_{0}-R_{0}+1\right)-\left(M_{1}-R_{1}+1\right), \\
& 1+Q_{3}=\left(M_{0}-R_{0}+1\right)-\left(M_{1}-R_{1}+1\right)+\left(M_{2}-R_{2}+1\right), \\
& 1-Q_{4}=\left(M_{0}-R_{0}+1\right)-\left(M_{1}-R_{1}+1\right)+\left(M_{2}-R_{2}+1\right)-\left(M_{3}-R_{3}+1\right), \\
& \cdot \cdot \cdot \cdot \cdot \cdot \cdot \cdot \cdot \cdot \cdot \cdot \cdot \cdot \cdot \cdot \cdot \cdot \cdot \cdot \cdot \cdot \cdot \cdot \cdot \cdot \cdot \cdot \cdot \cdot \cdot \\
& 1+(-1)^{n-1} Q_{n}=\left(M_{0}-R_{0}+1\right)-+\cdot \cdot+(-1)^{n-1}\left(M_{n-1}-R_{n-1}+1\right) .
\end{aligned}
$$

Of the equations of Theorem 7 which are not included in (159) there remain the equations

$$
\begin{aligned}
R_{n}-1 & =0, \\
M_{n} & =Q_{n} .
\end{aligned}
$$


With the aid of (161) we can write

$$
Q_{n}=M_{n}-R_{n}+1 \text {. }
$$

If this expression for $Q_{n}$ be substituted for $Q_{n}$ in the last of equations (160) there will result the equality given by the last of the equations of Theorem 8 The remaining relations of Theorem 8 result immediately from (160) upon recalling that the $Q$ 's are all either positive or zero. Thus the theorem is proved.

If the domain $S$ be one which can in particular be put into one-to-one continuous correspondence with the points in $n$-space interior to and on an (n-1)-dimensional hypersphere, then the connectivity numbers $R_{i}$ of $S$ are all unity. Hence we have the following corollary to Theorem 8 .

COROLlaRY. If $S$ be an $n$-dimensional region homeomorphic with the points in $n$-space interior to and on an $(n-1)$-dimensional hypersphere, and $\boldsymbol{M}_{i}$ be the number of critical points of the ith type of $f$ in $S(i=0,1, \ldots, n)$ then under the boundary conditions $\alpha$ the following relations hold true:

$$
\begin{aligned}
& 1 \leqq M_{0}, \\
& 1 \geqq M_{0}-M_{1}, \\
& 1 \leqq M_{0}-M_{1}+M_{2}, \\
& 1 \geqq M_{0}-M_{1}+M_{2}-M_{3}, \\
& \cdot \cdot \cdot \cdot \cdot \cdot \cdot \cdot \cdot \cdot \cdot \cdot \cdot \\
& 1 \leqq\left[M_{0}-M_{1}+M_{2}-M_{3}+-\ldots+(-1)^{n-1} M_{n-1}\right](-1)^{n-1}, \\
& 1=M_{0}-M_{1}+M_{2}-M_{3}+-\ldots+(-1)^{n} M_{n} .
\end{aligned}
$$

20 . The boundary conditions $\beta$ defined. The boundary conditions $\beta$ are the following.

I. The boundary $B$ of the domain $R$ of $\S 1$ shall consist of a closed set of points lying in a finite part of the space of the variables $\left(x_{1}, x_{2}, \ldots, x_{n}\right)$.

II. The points on $B$ in the neighborhood of any particular point $\left(X_{0}\right)$ on $B$ shall satisfy a relation of the sort

$$
F\left(x_{1}, x_{2}, \cdots, x_{n}\right)=0
$$

where $F\left(x_{1}, x_{2}, \cdots, x_{n}\right)$ is a single-valued continuous function of $\left(x_{1}, x_{2}, \cdots, x_{n}\right)$ possessing continuous first, second, and third partial derivatives in the neighborhood of each point of $B$, of which not all of the first partial derivatives are to be zero at any point of $B$.

III. The function $f\left(x_{1}, x_{2}, \cdots, x_{n}\right)$ in addition to satisfying the conditions of $\S 1$ shall be continuous on $B$, and its first partial derivatives shall take on continuous boundary values on $B$. 
IV. At each point of $B$ the unilateral directional derivative of $f\left(x_{1}, x_{2}, \cdots, x_{n}\right)$ along the normal to $B$ in the sense that leads from points of $R$ to points not in $R$, and on the side that lies in $R$, shall be positive.

21 . The boundary conditions $\beta$ reduced to the boundary conditions $a(\S 4)$. By a redefinition of $f^{\prime}\left(x_{1}, x_{2}, \ldots, x_{n}\right)$ in the neighborhood of $B$ there can be obtained a function $L\left(x_{1}, x_{2}, \ldots, x_{n}\right)$ with critical points identical in position and in type with those of $f^{\prime}\left(x_{1}, x_{2}, \ldots, x_{n}\right)$, but such that one of the manifolds

$$
L\left(x_{1}, x_{2}, \cdots, x_{n}\right)=\text { const. }
$$

will serve as a boundary $A$ satisfying the earlier boundary conditions.

Under condition II of the boundary conditions $\beta$ there is a definite normal to $B$ at each point $\left(x_{1}^{0}, x_{2}^{0}, \cdots, x_{n}^{0}\right)=\left(X_{0}\right)$ of $B$ whose equations may be given in the form

$$
x_{i}-x_{i}^{0}=A_{i}\left(x_{1}^{0}, x_{2}^{0}, \ldots, x_{n}^{0}\right) s \quad(i=1,2, \ldots, n),
$$

where $A_{i}\left(x_{1}^{0}, x_{2}^{n}, \cdots, x_{n}^{0}\right)$ together with its first and second partial derivatives are continuous functions of their arguments in the neighborhood of any given point on $B$, where $s$ is the distance along the normal, being zero on $B$, and increasing as the point on the normal crosses $B$ from points of $R$ to points not in $R$. We can and will suppose $s_{1}$ to be a negative constant chosen so small that the points $\left(x_{1}, x_{2}, \ldots, x_{n}\right)=(X)$ on the normal (162) at the points at which

$$
s_{1} \leqq s<0
$$

include once and only once every point of $R$, not including $B$, in the neighborhood of $B$.

Because of the boundary conditions IV we can and will suppose that $s_{1}$ is so small that the points $(X)$ given by (162) for values of $s$ satisfying (163) contain no critical points of $f$. It follows that there is one and only one of the trajectories orthogonal to the manifolds $f=$ const., through each point of (162) for which (163) holds. Let $s_{z}, s_{3}$, and $s_{4}$ be three constants such that

$$
s_{1}<s_{2}<s_{3}<s_{4}<0 .
$$

Let $T_{1}, T_{2}, T_{3}$, and $T_{4}$ be respectively the $(n-1)$-dimensional manifolds of points $(X)$ obtained by putting $s=s_{1}, s_{z}, s_{3}$, and $s_{4}$ in (162) and letting $\left(X_{0}\right)$ vary on $B$. It is a consequence of boundary conditions IV, as the calculation of the appropriate jacobian will show, that if $s_{1}$ be a sufficiently small negative constant, any trajectory orthogonal to the manifolds $f^{\prime}=$ const. passing through a point $P_{1}$ of $T_{1}$ also passes through 
uniquely determined points $P_{2}, P_{3}$, and $P_{4}$ on $T_{2}, T_{3}$, and $T_{4}$, respectively, such that the positions of $P_{2}, P_{3}$, and $P_{4}$ are continuous functions of the position of $P_{1}$ on $T_{1}$.

We again make use of that representation of the orthogonal trajectories in which the parameter at any point equals the value of $f$ at that point, and we let $\tau_{1}, \tau_{2}, \tau_{3}$, and $\tau_{4}$ respectively be the values of $\tau$ at $P_{1}, P_{2}$, $P_{3}$ and $P_{4}$.

We now are in a position to replace $f\left(x_{1}, x_{2}, \ldots, x_{n}\right)$ by a new function $L\left(x_{1}, x_{2}, \ldots, x_{n}\right)$ defined as follows. At each point of $R$ not a point given by (162) for a value of $s>s_{2}$, we define

$$
L\left(x_{1}, x_{2}, \ldots, x_{n}\right) \equiv f\left(x_{1}, x_{2}, \ldots, x_{n}\right) .
$$

To define $L\left(x_{1}, x_{2}, \ldots, x_{n}\right)$ further it will be convenient, for those points $(X)$ given by (162) for which

$$
s_{1} \leqq s \leqq s_{4},
$$

to replace $\left(x_{1}, x_{2}, \ldots, x_{n}\right)$ by a new set of $n$ independent variables, namely $(n-1)$ independent parameters $\left(u_{1}, u_{2}, \ldots, u_{n-1}\right)$, determining a point $P_{1}$ on $T_{1}$ and thereby a trajectory through $P_{1}$, and an $n$th independent parameter, namely the above parameter $\tau$ determining a point $(X)$ on the trajectory through $P_{1}$. To represent a set of points $(X)$ satisfying (165) and neighboring a particular trajectory, the parameters $\left(u_{1}, u_{2}, \ldots, u_{n-1}\right)$ can be so chosen that the coördinates of $(X)$ are singlevalued functions of the variables $\left(u_{1}, u_{2}, \ldots, u_{n-1}, \tau\right)$ provided with continuous second partial derivatives. Now in terms of the new variables the points satisfying (165) are the points at which

$$
\tau_{1} \leqq \tau \leqq \tau_{4} \text {. }
$$

Of these points the points at which

$$
\tau_{1} \leqq \tau \leqq \tau_{2}
$$

are points at which $L\left(x_{1}, x_{2}, \ldots, x_{n}\right)$ has already been defined. Setting

$$
L\left(x_{1}, x_{2}, \ldots, x_{n}\right)=\varphi\left(\tau, u_{1}, u_{2}, \ldots, u_{n-1}\right)
$$

the previous definition of $L$, namely (164), reduces for the points of (167) to

$$
\varphi\left(\tau, u_{1}, u_{2}, \cdots, u_{n-1}\right) \equiv \boldsymbol{\tau} .
$$

To define $L\left(x_{1}, x_{2}, \ldots, x_{n}\right)$ further note that $\tau_{1}, \tau_{2}, \tau_{3}$, and $\tau_{4}$ are functions of $\left(u_{1}, u_{2}, \cdots, u_{n-1}\right)$. Let $G$ be a constant greater than the 
value of $f$ in $R$. For those points of (166) for which

we now define

$$
\tau_{2} \leqq \tau \leqq \tau_{4}
$$

$$
\varphi\left(\tau, u_{1}, u_{2}, \cdots, u_{n-1}\right) \equiv \boldsymbol{\tau}+H\left(u_{1}, \cdots, u_{n-1}\right)\left[\tau-\tau_{2}\left(u_{1}, \cdots, u_{n-1}\right)\right]^{4}
$$

where $H$ is defined by the equation

$$
G=\tau_{3}+H\left[\tau_{3}-\tau_{2}\right]^{4}
$$

as a positive function of $\left(u_{1}, u_{2}, \ldots, u_{n-1}\right)$. From (170) and (171) it follows that

$$
\varphi\left(\tau_{3}, u_{1}, u_{2}, \cdots, u_{n-1}\right)=G .
$$

For any point corresponding to a value of $\tau$ satisfying (169) we now define

$$
L\left(x_{1}, x_{2}, \cdots, x_{n}\right) \equiv \varphi\left(\tau, u_{1}, \cdots, u_{n-1}\right)
$$

at that point.

Let $R^{\prime}$ be the domain in which $L$ has been defined. $L\left(x_{1}, x_{2}, \ldots, x_{n}\right)$ and its second partial derivatives are readily seen to be continuous in $R^{\prime}$. No critical points are introduced by the definition (170), as follows upon verification of the fact that the partial derivative $\varphi_{\tau}\left(\tau, u_{1}, \ldots, u_{n-1}\right)>0$ for points for which (170) and (171) hold. Thus in the neighborhood of critical points of $f$ or of $L$, (164) holds. From (172) it follows that on the manifold $T_{3}$

$$
L\left(x_{1}, x_{2}, \ldots, x_{n}\right)=G .
$$

Boundary conditions $\alpha$ of $\S 4$ will obviously be satisfied if we take for the manifold $A$ used in boundary conditions $\alpha$ the manifold $T_{3}$. Further, with the aid of the normals (162) it is easy to see that the complex of points satisfying $L\left(x_{1}, x_{2}, \ldots, x_{n}\right) \leqq G$ can be put into one-to-one continuous correspondence with the complex consisting of the domain $R$ and the above boundary $B$. Thus these two complexes have the same connectivity numbers. From Theorem 8 we obtain accordingly the following theorem.

THEOREM 9. Under the boundary conditions $\beta$ the relations between the numbers $M_{i}$, where $M_{i}(i=0,1, \cdots, n)$ is the number of critical points of $f$ of type $i$ in $R$, and the numbers $R_{j}$, where $R_{j}(j=0,1, \ldots, n)$ is the jth connectivity number of the complex consisting of $R$ and its boundary $B$, are the same as those given in Theorem 8.

Cornell University, ITHACA, N. Y. 